\title{
Hydrochemical characteristics of the groundwater in Trans-Yamuna Alluvial aquifer, Palwal District, Haryana, India
}

\author{
Sarfaraz Ahmad ${ }^{1} \cdot$ Nepal Singh $^{1} \cdot$ Syeda Nigar Mazhar ${ }^{1}$
}

Received: 7 February 2018 / Accepted: 21 January 2020 / Published online: 11 February 2020

(c) The Author(s) 2020

\begin{abstract}
Hydrochemical processes have been evaluated for the groundwater regime in Trans-Yamuna alluvial aquifer, Palwal District, Haryana. The groundwater belongs to the $\mathrm{Na}^{+}-\mathrm{Mg}^{+2}$ and $\mathrm{Cl}^{-}-\mathrm{SO}_{4}^{-2}$ facies, and solute acquisition processes indicated that the rock weathering/evaporation is a major mechanism that characterizes the hydrochemistry. It is also indicated that dissolution and reverse ion exchange processes are playing a major role in determining the groundwater chemistry in the study area. The varied climate in Ganga plains is responsible for the importance of different hydrogeochemical processes that impact on hydrochemistry of groundwater. Groundwater quality parameters were tested for drinking and irrigation purposes. Water quality index shows that $13 \%, 31 \%, 34 \%$ and $21 \%$ fall in good, poor, very poor and unsuitable water category, respectively, for drinking purposes. On the basis of SAR and $\mathrm{Na} \%$, the groundwater indicated that it is safe for irrigation purposes. But high TDS of few samples suggested the salinity hazard while using it for irrigation purposes for a longer period.
\end{abstract}

Keywords Hydrochemical characteristics · Seasonal variations · Trans-Yamuna River

\section{Introduction}

The success of the green revolution resulted in unprecedented development in the state of Haryana in India. It was assisted by groundwater as a key source of irrigation that resulted in an increase in agriculture productivity exponentially. A general increase in wealth of population due to high agriculture productivity subsequently resulted in rural-urban migration, industrialization and economic development in the last 50 years in urban-rural areas of India. These activities are putting huge pressure on natural resources. A unique geological and climatic condition in the Trans-Yamuna area supported less development of the groundwater due to hydrometeorological and geological conditions. Therefore, less-water-required agricultural practices were adopted in this region. But in the last 50 years, groundwater is being

Sarfaraz Ahmad

sarf71@gmail.com

Nepal Singh

nepalsingh.amu@gmail.com

Syeda Nigar Mazhar

nigarmazhar.amu@gmail.com

1 Department of Geology, Aligarh Muslim University, Aligarh 202002, India continuously used to irrigate types of crops that need huge irrigation. It has resulted in environmental degradation in terms of groundwater and soil resources in the region.

During the last few decades, it is also observed that groundwater has been polluted drastically because of changes in agricultural expansion, industrialization, improper solid and toxic waste management practices throughout India (Ramesh and Soorya 2012; Kumar et al. $2009,2011)$. Numbers of studies on groundwater quality deterioration and geochemical evolution of groundwater have been carried out in different parts of India as well as in other parts of the world (Jalali 2006, Jalali 2007, Gupta et al. 2008; Irfan and Said 2008; Kumar et al. 2009; Srinivasamoorthy et al. 2010; Zhang et al. 2011; Vasanthavigar et al. 2012). It is found that several regions in India have encountered degradation in groundwater quality too due to an increase in population growth, change in land use/land cover and rapid urbanization (Brindha and Elango 2010; Ramesh and Elango 2005).

The present study area of Trans-Yamuna aquifer is characterized by high population growth, urbanization, industrialization and the rapid increase in agricultural productivity. The impact of such circumstances may adversely affect the future sustainability of the groundwater in the area. Therefore, the present study 
was carried out for the assessment of hydrogeochemical characteristics in the area facing environmental stresses due to groundwater overexploitation in recent years. The findings of this study will help to protect the quality of groundwater for the present and to prepare future groundwater for sustainable water supply in the region.

\section{Study area}

Palwal District of Haryana lies between $27^{\circ} 50^{\prime}: 28^{\circ} 15^{\prime} \mathrm{N}$ latitudes and $77^{\circ} 05^{\prime}: 77^{\circ} 33^{\prime} \mathrm{E}$ longitudes, covering an area of about $1364.55 \mathrm{Km}^{2}$ with an average elevation of $195 \mathrm{amsl}$ The district comprises river flood plains, low lying plains, depressions, sand dunes and hills. Geologically, the area is situated in the Yamuna sub-basin of the Ganga basin in India (Fig. 1).

\section{Climate}

This area shows tropical steppe, semiarid and arid climatic condition. The climate exhibits the following characteristics: (a) seasonal rhythm, (b) cool winter, (c) hot summer, (d) mostly dry weather except for two to three months (July to September), (e) unreliable rainfall (f) meager rainfall and (g) high annual range of temperature. The study area is very hot in summer (up to $49{ }^{\circ} \mathrm{C}$ ) and cold in winters (down to $\left.1{ }^{\circ} \mathrm{C}\right)$. The hottest months are May and June, and the coldest being December and January. With the onset of March, the temperature begins to increase rapidly and goes up to $49^{\circ} \mathrm{C}$ in May and June. From April onwards, hot dust-laden winds locally known as Loo blows and weather is unpleasant.

Rainwater resource plays an important role in every aspect of human activity. Rainfall is markedly restricted in season, and drought is a usual occurrence in this area.

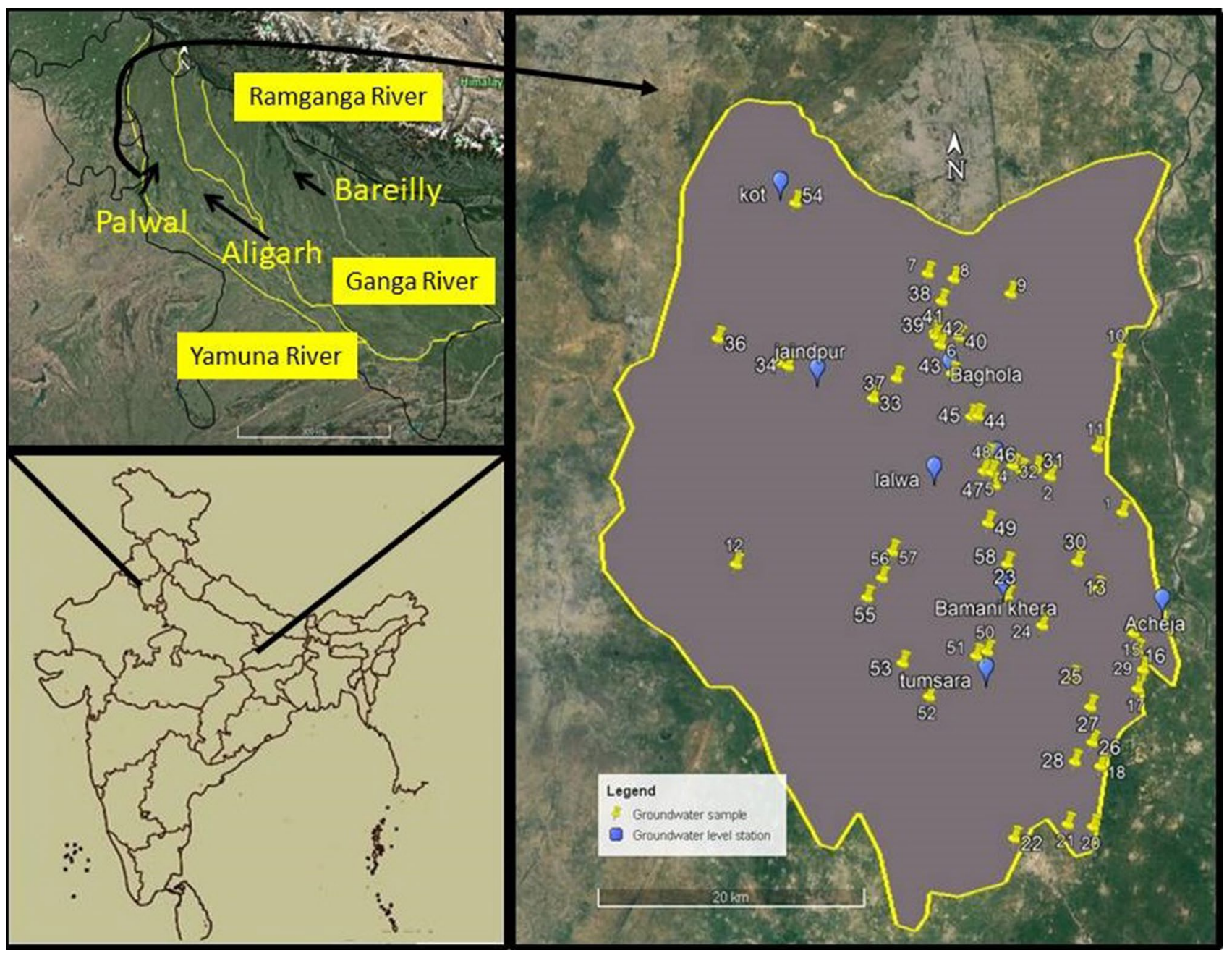

Fig. 1 Location map of the study area along with the sample locations 
Variation in rainfall from year to year is considerable. The distribution of rainfall is irregular in both time and space, and water deficiency is a problem in most of the area. About $80 \%$ of the rainfall occurs in the monsoon season (July to September) and sometimes causes local flooding. The annual rainfall including monsoon of the region is about $300-500 \mathrm{~mm}$ which is unevenly distributed over the area. During 3 months from the last week of June to September, the moist air of oceanic origin penetrates into the region and causes high humidity, cloudiness and monsoon rainfall.

\section{Geology}

The basement of the area is composed of quartzite and slate of Delhi Supergroup of Achaean age. It is overlain by sediments of older alluvium (upland unit of the area) of the Pleistocene age. Lowland areas near the flood plain are newer alluvium of Holocene age overlain on older alluvium. Newer alluvium is confined within floor plains of Yamuna River and disconformably overlies the older alluvium. The newer alluvium is devoid of calcareous nodules, while the older alluvium consists of calcareous Kankar. The northwest part of the area is covered by quaternary eolian sediment (Fig. 2).

The lithological character and water-bearing characteristics of formations are summarized as follows:

\begin{tabular}{lll}
\hline Geological age & Litho unit & Morpho unit \\
\hline (Quaternary) Holocene & Newer alluvium & Lowland unit \\
Quaternary (Eolian) & Disconformities & \\
(Quaternary) Pleistocene & Older alluvium & Up land unit \\
Unconformity Proterozoic & Delhi Supergroup & Denuded hill unit
\end{tabular}

(After CGWB 2006)

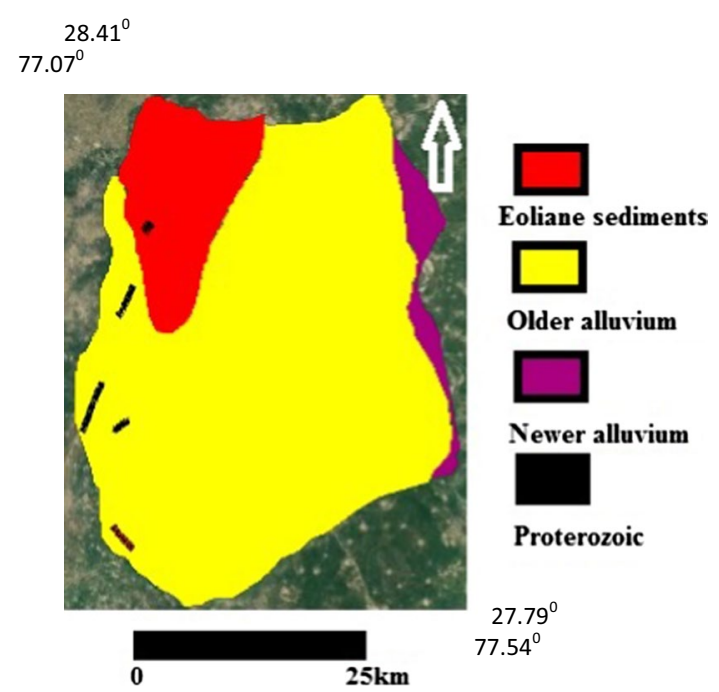

Fig. 2 Geology of the Trans-Yamuna region, Palwal District, Haryana

\section{Soil cover}

The alluvium and eolian soil are the main extensive cover area in the area. The texture of the soil is sand to loamy in recent Yamuna flood plains, sandy loam for older plains, sandy loam to clay loam for alluvial plains, and clay loam/ silty clay for low lying plains and depressions. The spatial distribution shows coarse loamy-calcareous soil in the northern part, fine loamy in southwest and calc-sandy in the eastern part, respectively (NBSS and LUP 1993).

\section{Hydrogeology}

The district is occupied by the Gangetic alluvial plain of Quaternary age and falls in the Yamuna sub-basin of the Ganga basin. The permeable granular zones are comprised of fine- to medium-grained sand and occasionally coarse sand and gravel. Groundwater occurs in alluvium and the underlying weathered/fractured quartzite. Alluvium comprises sand silt, Kankar and gravel, which form the principal groundwater-bearing horizon. In quartzite formation, occupying the northwestern part of the district, groundwater occurs in weathered and jointed fractured horizons (CGWB 2013). The depth to the water table in the area ranges from 2.00 to $20.75 \mathrm{mbgl}$ during the pre-monsoon period and from 2 to $9.40 \mathrm{mbgl}$ during the post-monsoon period and the average gradient of the water table is of the order of $1 \mathrm{~m} / \mathrm{km}$ with groundwater flow from north to south direction (Fig. 3).

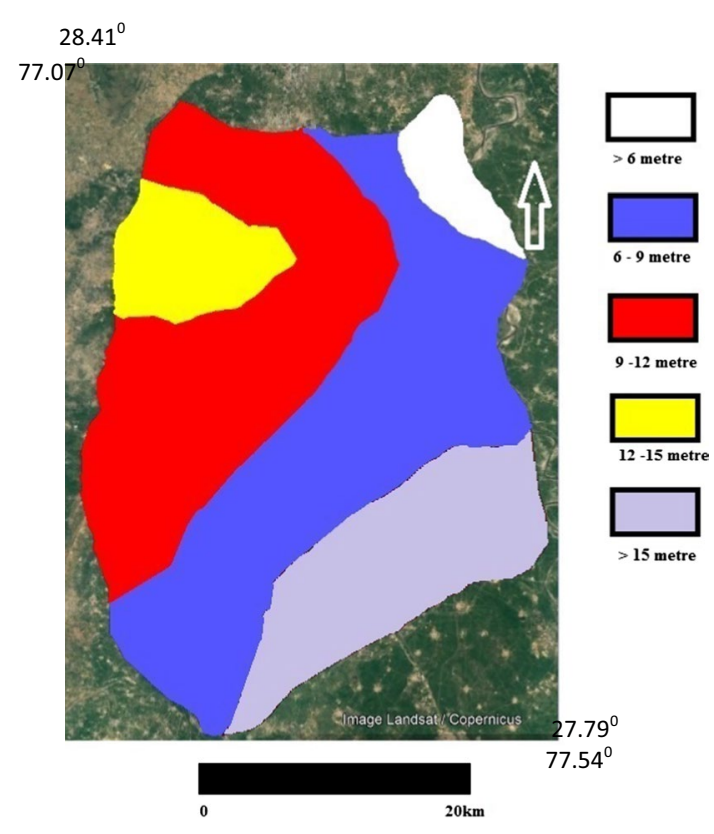

Fig. 3 Spatial distribution of the average groundwater depth in TransYamuna region, Palwal District

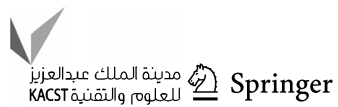




\section{Land use and land cover}

The land use pattern broadly constitutes built-up land as a habitat, agriculture land, industrial land and water bodies, forest, scrubland, wasteland and urban land. The forest area covers 2197 ha, built-up area 7436 ha, agriculture land 130,056 ha, wasteland 1344 ha, water bodies 1673 and other 369 ha. A small number of water bodies are considered in Silauthi, Aurangabad, Sholaka and Dighaut, while the mining areas are found in Sarai, Tumsara and Sugar mill side (Dept. of Town and Country Planning 2013).

\section{Methodology}

The present study is designed to evaluate the hydrogeochemical processes that are responsible for hydrogeochemical characteristics for the groundwater in Trans-Yamuna alluvial plain, Palwal District, Haryana. The grid sampling method was adopted to collect the samples from the study area, and the samples were collected in post-monsoon 2015 and premonsoon 2016. Hydrochemical parameters, i.e., pH, EC (electrical conductivity) and concentrations of $\mathrm{Ca}^{+2}, \mathrm{Mg}^{+2}$, $\mathrm{Na}^{+}, \mathrm{K}^{+}, \mathrm{Cl}^{-}, \mathrm{HCO}_{3}^{-}, \mathrm{Cl}^{-}, \mathrm{SO}_{4}^{-2}$ and $\mathrm{NO}_{3}{ }^{-}$, in groundwater were determined. The groundwater samples were collected from hand-pumps-fitted boreholes at different locations of Palwal District, Haryana. About 30-50 L of water was drawn from hand pumps before collecting the samples in plastic bottles and analyzed as per standard method (APHA 1998) and the summary of these methods is presented in Table 1. The groundwater depth data were downloaded from IndiaWRIS Web GIS Web site, and the rates of change in groundwater depth in $\mathrm{m} /$ year were determined using groundwater depth time series data from 1994 to 2016. Spatial and temporal coverage of the settlement area has been downloaded for the Palwal City from Bhuvan ISRO's Geoportal. The growth of the settlement area has been determined using PCI Geomatica 9.1. Population data of Palwal District for the periods 1991 and 2011 were obtained from India Census. The comparative analysis has been conducted using the hydrochemical data for Bareilly District from Ahmad and Mazhar (2020) and data from the Aligarh Doab region from Wasim et al. (2014).

\section{Results and discussion}

The details of the parameters for the groundwater samples in pre-monsoon and post-monsoon period and the average of these parameters are presented in Tables 2, 3 and 4. A comprehensive observation is as follows: $\mathrm{pH}$ value $8 \pm 0.28$ (7.4-8.4), $\mathrm{HCO}_{3}{ }^{-} 384 \pm 208(49-1281) \mathrm{mg} / \mathrm{l}$, $\mathrm{Mg}^{+2} 184 \pm 22(455-103 \mathrm{mg} / \mathrm{l})$ and $\mathrm{SO}_{4}{ }^{2-} 423 \pm 509$ (2183-9) $\mathrm{mg} / \mathrm{l}$. $\mathrm{Cl}^{-}$shows $776 \pm 668$ (3302-105) $\mathrm{mg} / \mathrm{l}$,
Table 1 Detailed methodology used in determining the hydrochemical characteristics of the groundwater

\begin{tabular}{|c|c|c|}
\hline Parameter & Instrument and reagents & References \\
\hline \multicolumn{3}{|l|}{ General } \\
\hline $\mathrm{pH}$ & $\mathrm{pH}$ meter (pH 4 and 9.2) & APHA (1998) \\
\hline $\mathrm{EC}$ & $\mathrm{EC}$ meter $(\mathrm{KCl})$ & APHA (1998) \\
\hline TDS & Heating at $180^{\circ} \mathrm{C}$, laboratory & APHA (1998) \\
\hline $\mathrm{TH}$ & $\begin{array}{l}\text { EDTA titrimetric (EDTA, ammonia buffer and Eriochrome Black T } \\
\text { (EBT) indicator) }\end{array}$ & APHA (1998) \\
\hline \multicolumn{3}{|c|}{ Major cations } \\
\hline $\mathrm{Ca}^{2+}$ & EDTA titrimetric (EDTA, sodium hydroxide and murexide) & APHA (1998) \\
\hline $\mathrm{Mg}^{2+}$ & $\begin{array}{l}\text { Calculation }(\mathrm{MgH}=\mathrm{TH}-\mathrm{CaH} ; \mathrm{Mg}=\mathrm{MgH} \text { X Eq. Wt of } \mathrm{Mg} \text { X normality } \\
\text { of EDTA) }\end{array}$ & APHA (1998) \\
\hline $\mathrm{Na}^{+}$ & Flame photometric $(\mathrm{NaCl})$ & APHA (1998) \\
\hline $\mathrm{K}^{+}$ & Flame photometric $(\mathrm{KCl})$ & APHA (1998) \\
\hline \multicolumn{3}{|c|}{ Major anions } \\
\hline $\mathrm{HCO}_{3}^{-}$ & Titrimetric $\left(\mathrm{H}_{2} \mathrm{SO}_{4}\right.$, phenolphthalein and methyl orange $)$ & APHA (1998) \\
\hline $\mathrm{Cl}^{-}$ & Titrimetric, silver nitrate $\left(\mathrm{AgNO}_{3}\right)$, potassium chromate & APHA (1998) \\
\hline $\mathrm{F}^{-}$ & Spands $(\mathrm{NaF})$ & APHA (1998) \\
\hline $\mathrm{SO}_{4}^{2-}$ & $\begin{array}{l}\text { Turbidity-UV-visible spectrophotometer method }(\mathrm{HCl} \text {, ethyl alcohol, } \\
\mathrm{NaCl} \text {, barium chloride, sodium sulfate) }\end{array}$ & APHA (1998) \\
\hline
\end{tabular}


and the $\mathrm{Ca}^{2+}$ concentration is $102 \pm 103 \mathrm{mg} / \mathrm{l}(890-24)$ $\mathrm{mg} / \mathrm{l}$. The $\mathrm{Na}^{+}$concentration is $337 \pm 355(1716-19) \mathrm{mg} / \mathrm{l}$, whereas the $\mathrm{K}^{+}$concentration is $81 \pm 355(263-14) \mathrm{mg} / \mathrm{l}$. It is found that monsoonal activity resulting in recharge of groundwater reflected in a decrease in TDS, $\mathrm{pH}$ and $\mathrm{Na}^{+}$values and increase in $\mathrm{K}^{+}, \mathrm{HCO}_{3}{ }^{-}$and $\mathrm{NO}_{3}{ }^{-}$due to leaching. The order of anions is characterized by $\mathrm{Cl}^{-}>\mathrm{SO}_{4}^{-2}>\mathrm{HCO}_{3}^{-}>\mathrm{F}^{-}$and $\mathrm{Cl}^{-}>\mathrm{HCO}_{3}{ }^{-}>\mathrm{SO}_{4}^{-2}>\mathrm{F}^{-}$, and the anions were in the order of $\mathrm{Na}^{+}>\mathrm{Mg}^{+2}>\mathrm{Ca}^{+2}>\mathrm{K}^{+}$ and $\mathrm{K}^{+}>\mathrm{Mg}^{+2}>\mathrm{Ca}^{+2}>\mathrm{Na}^{+}$in pre- and post-monsoon periods, respectively.

The Piper plot of chemical data on a diamondshaped trilinear diagram reveals that the majority of groundwater samples fall in the fields of 3 and 4, i.e., $\mathrm{Na}^{+}-\mathrm{Mg}^{+2}-\mathrm{Cl}^{-}-\mathrm{SO}_{4}^{-2}$ type, suggesting that alkaline exceeds in most of the water samples. However, few groundwater samples fall in no-dominant type (Fig. 4). A comparative analysis has been conducted for a water sample from different parts of the Ganga-Yamuna aquifer. It indicated that the groundwater facies of the Ramganga aquifer in the Bareilly District show dramatically changes with seasons. But, the groundwater facies from Doab (interfluves) aquifer of Ganga-Yamuna and Trans-Yamuna aquifer do not show any significant change with seasons (Fig. 5). It suggests that the high permeability of aquifer material and slope facilitates the flushing of old groundwater from the Ramganga aquifer and causes a significant change in groundwater chemistry during the post-monsoon season. But a change in hydrochemical characteristic has not been indicated by the groundwater samples from Palwal and Aligarh districts.

An assessment of the relative importance of different hydrochemical processes was performed in Ramganga-Ganga-Yamuna aquifer area using Durov diagram (Fig. 6). The diagram indicates a systematic change in different hydrochemical processes identified from NE to SW in the Ganga basin. The ion exchange process was dominant in Ramganga aquifer; dissolution action was prominent in Ganga-Yamuna Doab region. But in Trans-Yamuna aquifer, most of the samples are indicating dissolution and few samples are exhibiting a reverse ion exchange as a dominant process. The northeast region is characterized by high rainfall and less evapotranspiration, while in the south on the present study area the climate becomes more arid. It is also revealed by a continuous change in hydrochemical processes that are reflected in a change in hydrogeochemical characteristics from NE to SW.

The base-exchange processes can be determined through base-exchange index $\mathrm{R}_{1}$. (Soltan 1999) and computed using
Eq. 1. It reveals the dominant processes and classifies the groundwater as $\mathrm{Na}^{+}-\mathrm{HCO}_{3}{ }^{-}$and $\mathrm{Na}^{+}-\mathrm{SO}_{4}^{-2}$ types if $\mathrm{R}_{1}<1$ or $>1$, respectively. In the present study, it is found that most of the samples are $\mathrm{Na}^{+}-\mathrm{HCO}_{3}{ }^{-}$type except one sample. Meteoric genesis index $R_{2}$ can be used to determine whether the sources of groundwater samples are deep meteoric water percolation type or shallow meteoric water percolation type. If $R_{2}<1$, the sources of groundwater are of deep meteoric water percolation type. If $R_{2}>1$, it shows the sources of groundwater are shallow meteoric water percolation type. Meteoric genesis index $R_{2}$ can be computed by using Eq. 2 . The results indicated that most of the groundwater samples fall under deep meteoric water percolation type. The semiarid climate along with the continuous overexploitation of groundwater resulted in steep fall in groundwater levels, which leads to deep meteoric percolation as a source of groundwater.

$R_{1}=\mathrm{Na}^{+}-\mathrm{Cl}^{-} / \mathrm{SO}_{4}^{-2}$ where all values are in meq $/$.

$R_{2}=\left(\mathrm{K}^{+}+\mathrm{Na}^{+}\right)-\mathrm{Cl}^{-} / \mathrm{SO}_{4}^{-2}$ where all values are in meq $/$ l.

\section{Sources of solute in groundwater}

Gibbs (1970) proposed the boomerang envelope model to describe the dynamics of chemical compositions of water and classified the controlling factors into three types, namely atmospheric precipitation, rock weathering and evaporation. The weight ratio $\mathrm{Na}^{+} /\left(\mathrm{Na}^{+}+\mathrm{Ca}^{+2}\right)$ as a function of TDS has been plotted (Fig. 7). It is inferred from the figure that all samples belong to rock weathering and moderate evaporation processes.

The sources and mechanisms controlling groundwater chemical composition have been further evaluated by applying the mass balance approach through scatter diagrams (Stallard and Edmond 1983). Analysis based on stoichiometric relationships demonstrates that in the present groundwater, $\mathrm{Ca}^{+2}+\mathrm{Mg}^{+2}$ together accounts for $47 \%$ of the total cations. The plot of $\mathrm{Ca}^{+2}+\mathrm{Mg}^{+2}$ versus $\mathrm{HCO}_{3}{ }^{-}$illustrates that equal numbers of groundwater samples show position above and below the 1:1 line of $\mathrm{Ca}^{+2}+\mathrm{Mg}^{+}$and $\mathrm{HCO}_{3}{ }^{-}$ion concentration. It implies that the part of $\mathrm{Ca}^{+2}$ and $\mathrm{Mg}^{+2}$ could be through silicate and carbonate weathering. Furthermore, moderate $\left(\mathrm{Ca}^{+2}+\mathrm{Mg}^{+2}\right) /\left(\mathrm{Na}^{+}+\mathrm{K}^{+}\right)$and $\left(\mathrm{Na}^{+}+\mathrm{K}^{+}\right) / \mathrm{Tz}^{+}$ equivalent ratio indicates that the mixed type (silicate and carbonate weathering) could be a major source of dissolved ions in the groundwater in the area (Fig. 8). 
Table 2 Hydrochemical characteristics of the groundwater in the Trans-Yamuna aquifer in post-monsoon, Palwal District (2015)

\begin{tabular}{|c|c|c|c|c|c|c|c|c|c|c|c|c|c|c|}
\hline S. no. & $\mathrm{EC}$ & $\mathrm{TH}$ & $\mathrm{pH}$ & $\mathrm{HCO}_{3}^{-}$ & $\mathrm{SO}_{4}^{2-}$ & $\mathrm{NO}_{3}^{-}$ & $\mathrm{Cl}^{-}$ & F- & $\mathrm{Ca}^{+2}$ & $\mathrm{Mg}^{+2}$ & $\mathrm{Na}^{+}$ & $\mathrm{K}^{+}$ & TDS & Er\% \\
\hline 1 & 0.85 & 0.3 & 7.5 & 377 & 140 & 6.855 & 438 & 0.5 & 54 & 121 & 44 & 152 & 1.3 & 8 \\
\hline 2 & 0.84 & 0.2 & 7.4 & 754 & 54 & 4.135 & 161 & 1.6 & 26 & 64 & 73 & 168 & 1.3 & 10 \\
\hline 3 & 0.64 & 0.3 & 7.1 & 195 & 70 & 3.756 & 449 & 0.6 & 45 & 105 & 39 & 86 & 1.0 & 8 \\
\hline 4 & 0.95 & 0.5 & 7.9 & 533 & 341 & 147.9 & 72 & 0.7 & 35 & 205 & 29 & 116 & 1.5 & -1 \\
\hline 5 & 0.79 & 2.8 & 7.6 & 377 & 137 & 0.589 & 228 & 0.5 & 96 & 84 & 77 & 228 & 1.2 & -10 \\
\hline 6 & 1.72 & 2.0 & 7.1 & 273 & 10 & 25.4 & 1368 & 1.2 & 237 & 55 & 126 & 584 & 2.7 & 9 \\
\hline 7 & 1.56 & 0.7 & 7.6 & 663 & 1 & 3.087 & 1152 & 1.2 & 32 & 327 & 10 & 240 & 2.4 & 10 \\
\hline 8 & 0.76 & 0.2 & 7.2 & 338 & 34 & 19.08 & 426 & 1.1 & 48 & 62 & 44 & 216 & 1.2 & 10 \\
\hline 9 & 0.32 & 0.1 & 6.9 & 169 & 31 & 11.84 & 133 & 0.3 & 55 & 19 & 3 & 75 & 0.5 & 9 \\
\hline 10 & 0.40 & 0.0 & 7.5 & 169 & 113 & 8.966 & 150 & 0.8 & 58 & 71 & 20 & 40 & 0.6 & -4 \\
\hline 14 & 1.37 & 0.7 & 6.9 & 364 & 143 & 7.916 & 864 & 0.7 & 289 & 310 & 29 & 132 & 2.1 & -10 \\
\hline 15 & 0.57 & 0.2 & 7.8 & 366 & 110 & 5.291 & 167 & 1.7 & 38 & 60 & 3 & 144 & 0.9 & 10 \\
\hline 16 & 1.24 & 2.1 & 7.1 & 299 & 158 & 1.703 & 835 & 1.3 & 96 & 146 & 44 & 364 & 1.9 & 7 \\
\hline 18 & 0.67 & 0.2 & 7.6 & 546 & 14 & 8.715 & 249 & 1.4 & 48 & 74 & 13 & 100 & 1.1 & 8 \\
\hline 21 & 2.03 & 1.0 & 6.9 & 546 & 70 & 105.1 & 1613 & 0.6 & 240 & 442 & 26 & 132 & 3.2 & 5 \\
\hline 22 & 1.22 & 0.6 & 6.7 & 442 & 88 & 3.28 & 864 & 0.8 & 64 & 246 & 27 & 176 & 1.9 & 7 \\
\hline 23 & 1.00 & 0.6 & 7.2 & 533 & 205 & 53.81 & 284 & 0.5 & 35 & 285 & 44 & 124 & 1.6 & -10 \\
\hline 24 & 1.34 & 0.4 & 7.4 & 611 & 485 & 17.14 & 497 & 1.4 & 38 & 193 & 29 & 228 & 2.1 & 10 \\
\hline 25 & 0.58 & 0.3 & 7.4 & 438 & 10 & 0.416 & 184 & 1.3 & 38 & 117 & 49 & 72 & 0.9 & -10 \\
\hline 26 & 0.91 & 0.3 & 7.6 & 676 & 157 & 5.264 & 249 & 1.4 & 28 & 138 & 18 & 152 & 1.4 & 11 \\
\hline 27 & 1.04 & 0.3 & 7.7 & 728 & 252 & 1.936 & 249 & 1.3 & 32 & 117 & 37 & 208 & 1.6 & 10 \\
\hline 29 & 1.03 & 0.6 & 7.2 & 455 & 117 & 0.327 & 475 & 0.7 & 320 & 144 & 20 & 72 & 1.6 & -10 \\
\hline 30 & 0.57 & 0.6 & 7.3 & 419 & 71 & 0.069 & 144 & 1.0 & 38 & 144 & 10 & 60 & 0.9 & -11 \\
\hline 31 & 0.48 & 0.3 & 7.0 & 286 & 78 & 2.302 & 158 & 0.7 & 74 & 111 & 10 & 24 & 0.7 & -10 \\
\hline 32 & 1.30 & 0.9 & 7.3 & 1040 & 52 & 13.76 & 426 & 0.1 & 29 & 402 & 8 & 60 & 2.0 & -9 \\
\hline 33 & 2.28 & 1.3 & 7.5 & 442 & 630 & 160.5 & 1368 & 1.3 & 106 & 573 & 27 & 256 & 3.6 & 3 \\
\hline 34 & 0.63 & 0.2 & 7.6 & 195 & 8 & 9.83 & 426 & 1.0 & 67 & 47 & 29 & 196 & 1.0 & 8 \\
\hline 36 & 0.85 & 0.6 & 7.5 & 442 & 36 & 0.273 & 461 & 1.6 & 42 & 249 & 18 & 75 & 1.3 & -9 \\
\hline 37 & 1.41 & 0.5 & 6.7 & 427 & 135 & 114.5 & 504 & 1.3 & 420 & 162 & 83 & 364 & 2.2 & -10 \\
\hline 38 & 1.10 & 0.6 & 7.1 & 260 & 51 & 9.503 & 746 & 0.8 & 80 & 216 & 42 & 308 & 1.7 & -8 \\
\hline 39 & 1.96 & 1.5 & 6.9 & 910 & 11 & 164.1 & 1136 & 1.1 & 48 & 123 & 488 & 184 & 3.1 & 10 \\
\hline 40 & 0.91 & 1.5 & 6.8 & 195 & 92 & 1.961 & 710 & 0.5 & 122 & 70 & 61 & 168 & 1.4 & 10 \\
\hline 41 & 0.98 & 0.8 & 7.8 & 273 & 531 & 1.626 & 284 & 1.6 & 38 & 53 & 224 & 124 & 1.5 & 10 \\
\hline 42 & 1.07 & 1.2 & 7.2 & 325 & 680 & 12.31 & 142 & 0.6 & 71 & 78 & 68 & 292 & 1.7 & 10 \\
\hline 43 & 1.40 & 2.0 & 7.2 & 377 & 102 & 12.29 & 1065 & 0.5 & 141 & 113 & 136 & 248 & 2.2 & 10 \\
\hline 44 & 1.07 & 0.5 & 7.5 & 507 & 37 & 22.58 & 634 & 1.0 & 29 & 207 & 27 & 208 & 1.7 & 5 \\
\hline 45 & 1.19 & 0.3 & 7.4 & 122 & 90 & 3.155 & 1065 & 1.1 & 100 & 115 & 92 & 268 & 1.9 & 10 \\
\hline 46 & 0.84 & 0.2 & 7.6 & 663 & 156 & 0.012 & 187 & 1.5 & 55 & 80 & 46 & 124 & 1.3 & 10 \\
\hline 47 & 0.97 & 0.7 & 7.0 & 366 & 128 & 23.11 & 497 & 0.5 & 96 & 277 & 46 & 86 & 1.5 & 10 \\
\hline 51 & 1.38 & 0.6 & 7.4 & 377 & 416 & 1.127 & 778 & 1.5 & 61 & 236 & 37 & 248 & 2.2 & 10 \\
\hline 52 & 0.53 & 0.3 & 7.1 & 0 & 81 & 1.19 & 446 & 0.6 & 77 & 47 & 20 & 152 & 0.8 & 8 \\
\hline 53 & 0.54 & 0.4 & 7.2 & 182 & 51 & 11.05 & 331 & 0.8 & 55 & 152 & 8 & 60 & 0.9 & -10 \\
\hline 54 & 1.03 & 0.5 & 7.3 & 325 & 178 & 7.875 & 663 & 1.4 & 106 & 187 & 13 & 124 & 1.6 & 7 \\
\hline 55 & 0.48 & 1.1 & 7.2 & 221 & 123 & 5.374 & 216 & 0.5 & 77 & 64 & 10 & 28 & 0.7 & 10 \\
\hline 56 & 1.34 & 0.8 & 7.3 & 468 & 111 & 0.468 & 781 & 1.7 & 87 & 60 & 61 & 520 & 2.1 & 10 \\
\hline 57 & 1.33 & 0.6 & 6.8 & 234 & 134 & 1.658 & 907 & 0.7 & 620 & 91 & 63 & 28 & 2.1 & 10 \\
\hline 58 & 0.92 & 1.0 & 7.2 & 403 & 377 & 2.386 & 288 & 0.9 & 97 & 96 & 46 & 124 & 1.4 & 10 \\
\hline Avg & 1.03 & 0.7 & 7.3 & 410.87 & 151 & 21.82 & 542 & 1.0 & 100 & 156 & 53 & 173 & 1.6 & 4 \\
\hline Max. & 2.28 & 2.8 & 7.9 & 1040 & 680 & 164.1 & 1613 & 1.7 & 620 & 573 & 488 & 584 & 3.6 & 11 \\
\hline Min. & 0.32 & 0.0 & 6.7 & 0 & 1 & 0.012 & 72 & 0.1 & 26 & 19 & 3 & 24 & 0.5 & -11 \\
\hline Std. & 0.59 & 0.6 & 0.3 & 205.05 & 162 & 42.48 & 383 & 0.4 & 113 & 114 & 76 & 118 & 0.7 & 9 \\
\hline
\end{tabular}

All the values are in ppm except EC and pH, EC ( $\mu \mathrm{S} / \mathrm{cm}$.), EC, TDS and TH in thousands 
Table 3 Hydrochemical characteristics of the groundwater in the Trans-Yamuna aquifer during pre-monsoon, Palwal District 2016

\begin{tabular}{|c|c|c|c|c|c|c|c|c|c|c|c|c|c|c|}
\hline S. no. & EC & TH & $\mathrm{pH}$ & $\mathrm{HCO}_{3}^{-}$ & $\mathrm{SO}_{4}^{2-}$ & $\mathrm{NO}_{3}^{-}$ & $\mathrm{Cl}^{-}$ & F- & $\mathrm{Ca}^{+2}$ & $\mathrm{Mg}^{+2}$ & $\mathrm{Na}^{+}$ & $\mathrm{K}^{+}$ & TDS & Er\% \\
\hline 1 & 1.01 & 0.4 & 8.6 & 286 & 152 & 4.2 & 568 & 0.8 & 35 & 144 & 345 & 44 & 1.6 & -10 \\
\hline 2 & 1.43 & 0.1 & 9.3 & 1456 & 3 & 3.3 & 128 & 0.6 & 29 & 35 & 496 & 76 & 2.2 & 0 \\
\hline 3 & 0.37 & 0.2 & 8.1 & 130 & 33 & 3.3 & 213 & 0.4 & 45 & 60 & 40 & 47 & 0.6 & -6 \\
\hline 4 & 1.25 & 0.3 & 8.9 & 390 & 277 & 19.5 & 710 & 0.5 & 38 & 123 & 312 & 80 & 2.0 & 9 \\
\hline 5 & 0.74 & 0.3 & 9.0 & 429 & 32 & 5.4 & 320 & 0.3 & 19 & 144 & 184 & 27 & 1.2 & -10 \\
\hline 6 & 0.66 & 0.3 & 9.0 & 260 & 75 & 5.1 & 355 & 0.6 & 19 & 115 & 180 & 16 & 1.0 & -8 \\
\hline 7 & 1.83 & 0.6 & 8.1 & 195 & 300 & 2.2 & 1287 & 0.8 & 513 & 94 & 200 & 270 & 2.9 & -3 \\
\hline 8 & 1.58 & 0.7 & 8.4 & 143 & 415 & 11.3 & 1136 & 0.5 & 96 & 304 & 332 & 37 & 2.5 & -2 \\
\hline 9 & 0.45 & 0.3 & 8.5 & 169 & 61 & 5.9 & 213 & 0.3 & 77 & 90 & 76 & 6 & 0.7 & -10 \\
\hline 10 & 0.75 & 0.4 & 8.5 & 156 & 147 & 4.3 & 355 & 0.3 & 58 & 160 & 272 & 21 & 1.2 & -10 \\
\hline 14 & 0.78 & 0.2 & 9.0 & 273 & 163 & 6.5 & 355 & 0.6 & 48 & 98 & 264 & 9 & 1.2 & -10 \\
\hline 15 & 0.78 & 0.2 & 8.9 & 273 & 165 & 6.5 & 350 & 0.6 & 48 & 98 & 260 & 15 & 1.2 & -10 \\
\hline 16 & 0.77 & 0.2 & 8.7 & 275 & 161 & 6.5 & 349 & 0.6 & 48 & 96 & 255 & 18 & 1.2 & -10 \\
\hline 18 & 1.41 & 1.0 & 8.2 & 130 & 511 & 9.9 & 710 & 0.4 & 160 & 390 & 256 & 30 & 2.2 & -10 \\
\hline 21 & 5.87 & 5.8 & 8.2 & 390 & 2516 & 72.9 & 3195 & 0.6 & 1106 & 427 & 1376 & 92 & 9.2 & 0 \\
\hline 22 & 1.82 & 0.4 & 9.1 & 585 & 272 & 67.7 & 639 & 0.5 & 32 & 160 & 1056 & 27 & 2.8 & -10 \\
\hline 23 & 1.06 & 0.3 & 9.0 & 390 & 488 & 28.4 & 255 & 0.5 & 38 & 133 & 296 & 25 & 1.7 & -2 \\
\hline 24 & 1.43 & 0.5 & 8.9 & 364 & 327 & 8.1 & 852 & 0.5 & 71 & 242 & 352 & 23 & 2.2 & -3 \\
\hline 25 & 0.63 & 0.3 & 9.0 & 312 & 97 & 2.3 & 213 & 0.5 & 19 & 134 & 164 & 49 & 1.0 & -10 \\
\hline 26 & 1.12 & 0.3 & 9.1 & 585 & 92 & 1.5 & 426 & 0.8 & 22 & 133 & 224 & 264 & 1.7 & -9 \\
\hline 27 & 1.13 & 0.3 & 9.1 & 377 & 363 & 2.5 & 497 & 0.6 & 48 & 127 & 312 & 35 & 1.8 & 2 \\
\hline 29 & 0.85 & 0.6 & 8.6 & 247 & 131 & 15.2 & 497 & 0.4 & 64 & 180 & 172 & 18 & 1.3 & -10 \\
\hline 30 & 0.72 & 0.4 & 8.5 & 427 & 89 & 2.3 & 213 & 0.5 & 64 & 144 & 144 & 40 & 1.1 & -10 \\
\hline 31 & 0.81 & 0.3 & 9.0 & 427 & 135 & 5.4 & 289 & 0.3 & 24 & 148 & 207 & 27 & 1.3 & -10 \\
\hline 32 & 1.08 & 0.3 & 8.8 & 392 & 39 & 18.3 & 695 & 0.5 & 22 & 125 & 310 & 80 & 1.7 & 1 \\
\hline 33 & 1.52 & 0.8 & 9.1 & 572 & 352 & 27.5 & 710 & 0.5 & 58 & 355 & 216 & 80 & 2.4 & -7 \\
\hline 34 & 1.08 & 0.3 & 8.4 & 0 & 400 & 13.4 & 497 & 0.7 & 80 & 542 & 144 & 3 & 1.7 & -10 \\
\hline 36 & 1.31 & 0.3 & 8.8 & 286 & 539 & 4.2 & 710 & 0.6 & 32 & 133 & 332 & 11 & 2.0 & 10 \\
\hline 37 & 3.61 & 0.5 & 9.2 & 897 & 1611 & 53.1 & 1420 & 0.6 & 48 & 201 & 1376 & 39 & 5.6 & 7 \\
\hline 38 & 3.62 & 0.5 & 9.1 & 896 & 1616 & 53.8 & 1413 & 0.6 & 45 & 221 & 1367 & 42 & 5.7 & 6 \\
\hline 39 & 6.44 & 3.6 & 8.3 & 195 & 2903 & 4.6 & 3905 & 0.6 & 80 & 302 & 2599 & 78 & 10.1 & 10 \\
\hline 40 & 1.84 & 0.9 & 8.5 & 366 & 635 & 2.9 & 852 & 0.4 & 71 & 405 & 529 & 14 & 2.9 & -10 \\
\hline 41 & 3.59 & 1.3 & 8.0 & 195 & 1837 & 0.6 & 1988 & 0.4 & 58 & 589 & 864 & 80 & 5.6 & 4 \\
\hline 42 & 3.60 & 0.5 & 9.1 & 903 & 1601 & 53.8 & 1410 & 0.6 & 48 & 200 & 1365 & 39 & 5.6 & 7 \\
\hline 43 & 0.87 & 0.4 & 8.7 & 208 & 421 & 3.0 & 312 & 0.4 & 74 & 150 & 172 & 15 & 1.4 & -5 \\
\hline 44 & 1.07 & 0.5 & 8.5 & 507 & 69 & 18.9 & 615 & 0.5 & 24 & 207 & 202 & 25 & 1.7 & 0 \\
\hline 45 & 0.91 & 0.3 & 8.2 & 0 & 9 & 2.7 & 994 & 0.5 & 26 & 110 & 260 & 20 & 1.4 & 10 \\
\hline 46 & 1.14 & 0.3 & 9.0 & 432 & 40 & 7.6 & 817 & 0.3 & 18 & 142 & 295 & 29 & 1.8 & 9 \\
\hline 47 & 1.24 & 0.3 & 8.9 & 455 & 238 & 13.1 & 680 & 0.5 & 26 & 122 & 319 & 79 & 1.9 & 8 \\
\hline 51 & 4.08 & 1.2 & 8.1 & 234 & 727 & 14.3 & 3195 & 0.4 & 603 & 119 & 1296 & 184 & 6.4 & 4 \\
\hline 52 & 1.33 & 0.6 & 8.5 & 611 & 167 & 22.6 & 710 & 0.5 & 64 & 244 & 248 & 14 & 2.1 & 0 \\
\hline 53 & 1.22 & 0.8 & 8.6 & 299 & 85 & 16.1 & 852 & 0.3 & 80 & 324 & 168 & 78 & 1.9 & -10 \\
\hline 54 & 1.30 & 1.0 & 8.5 & 208 & 99 & 5.0 & 994 & 0.6 & 128 & 390 & 200 & 9 & 2.0 & -10 \\
\hline 55 & 0.44 & 1.1 & 8.1 & 220 & 236 & 5.1 & 69 & 0.4 & 70 & 60 & 22 & 9 & 0.7 & 6 \\
\hline 56 & 0.92 & 0.8 & 9.0 & 466 & 221 & 0.4 & 210 & 1.3 & 84 & 21 & 368 & 59 & 1.4 & -10 \\
\hline 57 & 0.66 & 0.6 & 8.1 & 218 & 262 & 1.1 & 284 & 0.7 & 87 & 85 & 25 & 62 & 1.0 & 11 \\
\hline 58 & 0.83 & 1.0 & 9.0 & 400 & 315 & 2.3 & 275 & 0.8 & 91 & 84 & 118 & 8 & 1.3 & 11 \\
\hline Avg. & 1.55 & 0.7 & 8.7 & 375.09 & 456 & 13.7 & 803 & 0.5 & 99 & 189 & 438 & 50 & 2.4 & -5 \\
\hline Max. & 6.44 & 5.8 & 9.3 & 1456 & 2903 & 72.9 & 3905 & 1.3 & 1106 & 589 & 2599 & 270 & 10.1 & 0 \\
\hline Min. & 0.37 & 0.1 & 8.0 & 0 & 3 & 0.4 & 69 & 0.3 & 18 & 21 & 22 & 3 & 0.6 & 0 \\
\hline Std. & 1.33 & 0.9 & 0.4 & 258.44 & 652 & 17.8 & 807 & 0.2 & 184 & 128 & 500 & 57 & 2.1 & 0 \\
\hline
\end{tabular}

All the values are in ppm except $\mathrm{EC}$ and $\mathrm{pH}, \mathrm{EC}(\mu \mathrm{S} / \mathrm{cm}$.) EC, TDS and TH in thousands 
Table 4 Average hydrochemical characteristics of the groundwater in the TransYamuna aquifer, Palwal District

\begin{tabular}{|c|c|c|c|c|c|c|c|c|c|c|c|c|c|c|}
\hline S. no. & EC & $\mathrm{TH}$ & $\mathrm{pH}$ & $\mathrm{HCO}_{3}^{-}$ & $\mathrm{SO}_{4}{ }^{2-}$ & $\mathrm{NO}_{3}^{-}$ & $\mathrm{Cl}^{-}$ & F- & $\mathrm{Ca}^{+2}$ & $\mathrm{Mg}^{+2}$ & $\mathrm{Na}^{+}$ & $\mathrm{K}^{+}$ & TDS & Er\% \\
\hline 1 & 1.16 & 0.3 & 8.0 & 309 & 460 & 4.5 & 484 & 0.8 & 40 & 143 & 298 & 71 & 1.8 & 1 \\
\hline 2 & 1.29 & 0.1 & 8.4 & 1281 & 30 & 3.3 & 136 & 0.9 & 28 & 42 & 390 & 99 & 2.0 & 2 \\
\hline 3 & 0.43 & 0.2 & 7.6 & 146 & 60 & 3.2 & 249 & 0.4 & 45 & 72 & 40 & 57 & 0.7 & -2 \\
\hline 4 & 1.22 & 0.4 & 8.4 & 426 & 378 & 46.1 & 550 & 0.6 & 38 & 143 & 241 & 89 & 1.9 & 10 \\
\hline 5 & 0.81 & 1.5 & 8.3 & 416 & 93 & 4.0 & 287 & 0.4 & 38 & 161 & 185 & 77 & 1.3 & 10 \\
\hline 6 & 0.90 & 1.2 & 8.1 & 263 & 61 & 9.2 & 568 & 0.8 & 74 & 100 & 167 & 158 & 1.4 & -3 \\
\hline 7 & 1.76 & 0.7 & 7.9 & 312 & 226 & 2.2 & 1249 & 0.9 & 393 & 152 & 153 & 263 & 2.7 & 0 \\
\hline 8 & 1.52 & 0.5 & 7.8 & 192 & 329 & 12.2 & 1172 & 0.6 & 84 & 244 & 260 & 82 & 2.4 & 7 \\
\hline 9 & 0.42 & 0.2 & 7.7 & 169 & 62 & 6.8 & 192 & 0.3 & 71 & 72 & 58 & 23 & 0.7 & 10 \\
\hline 10 & 0.68 & 0.2 & 8.0 & 159 & 167 & 5.0 & 303 & 0.4 & 58 & 138 & 209 & 26 & 1.1 & 10 \\
\hline 14 & 0.95 & 0.5 & 8.0 & 296 & 194 & 6.4 & 479 & 0.6 & 108 & 151 & 205 & 40 & 1.5 & -10 \\
\hline 15 & 0.78 & 0.2 & 8.3 & 351 & 179 & 5.8 & 304 & 0.9 & 46 & 89 & 196 & 47 & 1.2 & -2 \\
\hline 16 & 0.91 & 1.2 & 7.9 & 281 & 199 & 5.0 & 468 & 0.8 & 60 & 109 & 202 & 105 & 1.4 & -2 \\
\hline 18 & 1.25 & 0.6 & 7.9 & 234 & 390 & 9.0 & 639 & 0.7 & 132 & 311 & 195 & 48 & 2.0 & -10 \\
\hline 21 & 4.92 & 3.4 & 7.6 & 429 & 1922 & 75.1 & 2794 & 0.6 & 890 & 431 & 1039 & 102 & 7.7 & 1 \\
\hline 22 & 1.68 & 0.5 & 7.9 & 549 & 248 & 49.3 & 692 & 0.6 & 40 & 181 & 799 & 64 & 2.6 & 10 \\
\hline 23 & 1.19 & 0.5 & 8.1 & 426 & 468 & 32.1 & 440 & 0.5 & 38 & 171 & 233 & 50 & 1.9 & 6 \\
\hline 24 & 1.53 & 0.5 & 8.1 & 426 & 488 & 9.5 & 834 & 0.7 & 63 & 230 & 271 & 74 & 2.4 & 7 \\
\hline 25 & 0.62 & 0.3 & 8.2 & 344 & 78 & 1.8 & 205 & 0.7 & 24 & 130 & 135 & 55 & 1.0 & 9 \\
\hline 26 & 1.13 & 0.3 & 8.4 & 608 & 148 & 2.2 & 437 & 1.0 & 24 & 134 & 173 & 236 & 1.8 & 0 \\
\hline 27 & 1.18 & 0.3 & 8.4 & 465 & 398 & 2.2 & 482 & 0.7 & 44 & 124 & 243 & 78 & 1.8 & 9 \\
\hline 29 & 0.94 & 0.6 & 7.9 & 299 & 156 & 11.0 & 490 & 0.5 & 128 & 217 & 134 & 32 & 1.5 & 10 \\
\hline 30 & 0.62 & 0.5 & 7.9 & 338 & 102 & 1.7 & 142 & 0.7 & 58 & 178 & 111 & 38 & 1.0 & 10 \\
\hline 31 & 0.74 & 0.3 & 8.0 & 392 & 115 & 4.3 & 256 & 0.4 & 36 & 138 & 180 & 26 & 1.1 & 10 \\
\hline 32 & 1.10 & 0.6 & 8.0 & 554 & 55 & 16.1 & 570 & 0.4 & 24 & 194 & 235 & 75 & 1.7 & -5 \\
\hline 33 & 1.80 & 1.0 & 8.3 & 540 & 579 & 54.6 & 870 & 0.7 & 70 & 409 & 169 & 124 & 2.8 & 0 \\
\hline 34 & 1.06 & 0.2 & 8.0 & 49 & 304 & 11.7 & 632 & 0.8 & 77 & 418 & 115 & 51 & 1.7 & -7 \\
\hline 36 & 1.20 & 0.4 & 8.2 & 325 & 422 & 3.1 & 646 & 0.8 & 34 & 162 & 254 & 27 & 1.9 & 10 \\
\hline 37 & 3.08 & 0.5 & 7.9 & 738 & 1227 & 63.0 & 1189 & 0.8 & 228 & 191 & 1053 & 120 & 4.8 & -1 \\
\hline 38 & 2.98 & 0.5 & 8.1 & 737 & 1238 & 40.6 & 1220 & 0.6 & 54 & 229 & 1036 & 109 & 4.7 & 4 \\
\hline 39 & 5.15 & 2.6 & 7.6 & 374 & 2183 & 39.0 & 3302 & 0.7 & 72 & 257 & 1716 & 105 & 8.0 & 9 \\
\hline 40 & 1.81 & 1.2 & 7.7 & 323 & 522 & 2.5 & 1008 & 0.5 & 83 & 322 & 508 & 53 & 2.8 & -9 \\
\hline 41 & 3.07 & 1.0 & 7.9 & 215 & 1643 & 0.8 & 1640 & 0.7 & 53 & 455 & 704 & 91 & 4.8 & 8 \\
\hline 42 & 3.25 & 0.9 & 8.2 & 759 & 1541 & 41.2 & 1377 & 0.6 & 54 & 170 & 1041 & 102 & 5.1 & 11 \\
\hline 43 & 1.09 & 1.2 & 7.9 & 250 & 366 & 4.8 & 607 & 0.4 & 91 & 141 & 163 & 73 & 1.7 & 8 \\
\hline 44 & 1.07 & 0.5 & 8.0 & 507 & 71 & 18.5 & 617 & 0.6 & 25 & 207 & 158 & 71 & 1.7 & 1 \\
\hline 45 & 1.23 & 0.3 & 7.8 & 124 & 9 & 2.6 & 1369 & 0.7 & 26 & 111 & 201 & 82 & 1.9 & 9 \\
\hline 46 & 1.01 & 0.3 & 8.3 & 490 & 108 & 5.4 & 551 & 0.6 & 27 & 126 & 224 & 53 & 1.6 & 6 \\
\hline 47 & 1.10 & 0.5 & 8.0 & 393 & 242 & 14.4 & 546 & 0.5 & 44 & 161 & 241 & 81 & 1.7 & -1 \\
\hline 51 & 3.47 & 0.9 & 7.8 & 270 & 753 & 10.5 & 2588 & 0.7 & 467 & 148 & 981 & 200 & 5.4 & 6 \\
\hline 52 & 1.14 & 0.4 & 7.8 & 458 & 165 & 16.5 & 643 & 0.5 & 67 & 194 & 191 & 49 & 1.8 & 1 \\
\hline 53 & 1.06 & 0.6 & 7.9 & 270 & 90 & 14.0 & 721 & 0.5 & 74 & 281 & 128 & 74 & 1.7 & 10 \\
\hline 54 & 1.26 & 0.7 & 7.9 & 237 & 163 & 5.3 & 909 & 0.8 & 123 & 339 & 153 & 38 & 2.0 & 11 \\
\hline 55 & 0.47 & 1.1 & 7.7 & 220 & 238 & 4.8 & 105 & 0.4 & 72 & 61 & 19 & 14 & 0.7 & 10 \\
\hline 56 & 1.17 & 0.8 & 8.2 & 467 & 221 & 0.4 & 527 & 1.4 & 85 & 22 & 328 & 174 & 1.8 & 5 \\
\hline 57 & 1.07 & 0.6 & 7.4 & 222 & 263 & 1.2 & 732 & 0.7 & 285 & 87 & 35 & 54 & 1.7 & 11 \\
\hline 58 & 0.97 & 1.0 & 8.1 & 401 & 526 & 2.2 & 277 & 0.8 & 92 & 80 & 91 & 37 & 1.5 & 11 \\
\hline Avg. & 1.47 & 0.7 & 8.0 & 384 & 423 & 14.6 & 776 & 0.6 & 102 & 184 & 337 & 81 & 2.3 & 4 \\
\hline Max. & 5.15 & 3.4 & 8.4 & 1281 & 2183 & 75.1 & 3302 & 1.4 & 890 & 455 & 1716 & 263 & 8.0 & 11 \\
\hline Min. & 0.42 & 0.1 & 7.4 & 49 & 9 & 0.4 & 105 & 0.3 & 24 & 22 & 19 & 14 & 0.7 & -10 \\
\hline Std. & 1.05 & 0.6 & 0.2 & 208 & 509 & 18.4 & 668 & 0.2 & 147 & 103 & 355 & 53 & 1.6 & 6 \\
\hline
\end{tabular}

All the values are in ppm except EC and pH, EC ( $\mu \mathrm{S} / \mathrm{cm}$.), TH, EC and TDS in thousands 
Fig. 4 Piper's diagram (Piper 1953) for pre- and post-monsoon groundwater

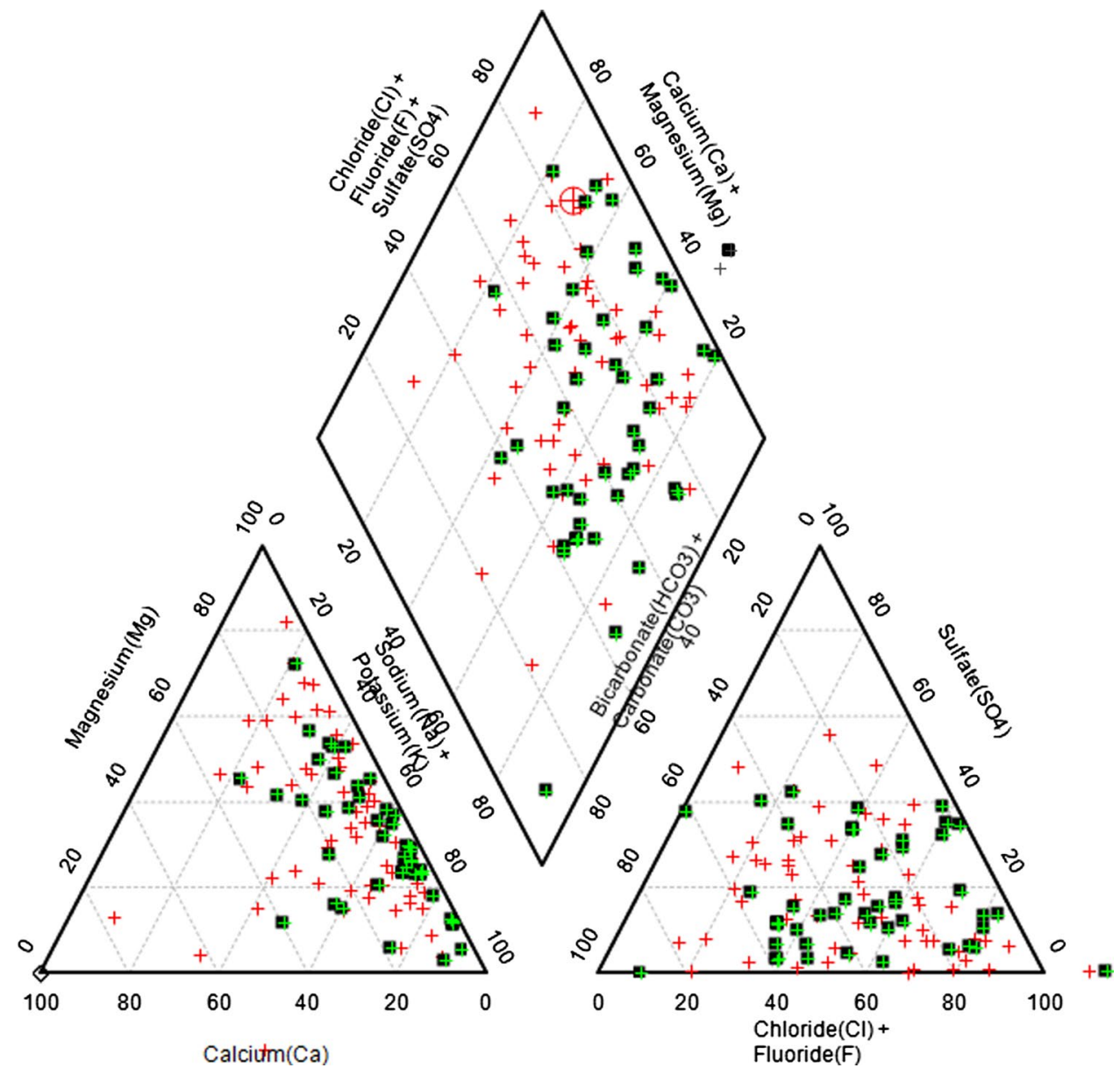

\section{Correlation matrix}

The statistical analysis has been carried out by correlation coefficient between different pairs of groundwater quality parameters of groundwater to assess the mechanism of solute acquisition processes in the area. The values of the coefficient of correlation were determined using SPSS software version 13 at probability $(p)$ of $<0.01$. Relationships during pre-monsoon and post-monsoon seasons showed positive and negative correlations among the parameters and significant relation are presented as bold numbers in the Table 5. The strong positive correlation of $\mathrm{HCO}_{3}{ }^{-}$with $\mathrm{Na}^{+}\left(R^{2}=0.77\right)$ and that of $\mathrm{Cl}^{-}$with $\mathrm{Na}^{+}\left(R^{2}=0.87\right)$ in premonsoon indicate the importance of the silicate weathering and evaporation. This relationship changes in post-monsoon due to recharge of the groundwater by monsoon rainfall; the strong positive correlation coefficient of $\mathrm{HCO}_{3}{ }^{-}$with $\mathrm{Na}^{+}$ $\left(R^{2}=0.71\right)$ and $\mathrm{Na}^{+}$with $\mathrm{Cl}^{-}\left(R^{2}=0.84\right)$ is maintained. The leaching of $\mathrm{SO}_{4}{ }^{2-}$ associated with $\mathrm{Mg}^{+2}$ and $\mathrm{Na}^{+}$is clearly indicated in the post-monsoon period. High input of $\mathrm{KNO}_{3}$ is being used as artificial fertilizer in the area, and leaching in the post-monsoon period shows a strong coefficient relationship $\left(R^{2}=0.61\right)$ between $\mathrm{K}^{+}$and $\mathrm{NO}_{3}^{-}$. A strong relationship between $\mathrm{Na}^{+}$and $\mathrm{Cl}^{-}$in the post-monsoon in groundwater indicates the dissolution of the salt which precipitated due to high evaporation in a dry climate and leaches during monsoonal recharge.

\section{Principal Component Analysis (PCA)}

The PCA technique has been used to recognize the important parameters that determine the water quality in many studies (Akbal et al. 2011; Varol and Davraz 2014). On investigating the existing water quality data using PCA, we attempt to find the governing factors that establish the quality of water by taking out important principal components (PCs). These components identify the processes that are associated with parameters showing the same behavior. These components explain $74 \%$ of the total variance in the pre-monsoon hydrochemical data and $70 \%$ of the total variance in the postmonsoon hydrochemical data (Table 6). The bold numbers in this table are showing significant contributions by the parameters towards the particular component.

During pre-monsoon, the first PC accounts for $36 \%$ of the total variance and it has a high correlation with $\mathrm{NO}_{3}{ }^{-}$, $\mathrm{Cl}^{-}, \mathrm{F}^{-}$, hardness, $\mathrm{Mg}^{+2}$ and TDS. The second component 
Fig. 5 Piper's diagram (Piper 1953) for groundwater in Bareilly, Palwal and Aligarh districts
Fig. 6 Durov diagram for groundwater in Bareilly, Palwal and Aligarh districts

\begin{tabular}{|c|c|}
\hline \multicolumn{2}{|c|}{ INDEX } \\
\hline 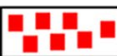 & Aligarh \\
\hline $4+4$ & Bareilly \\
\hline 0000 & Palwal \\
\hline
\end{tabular}
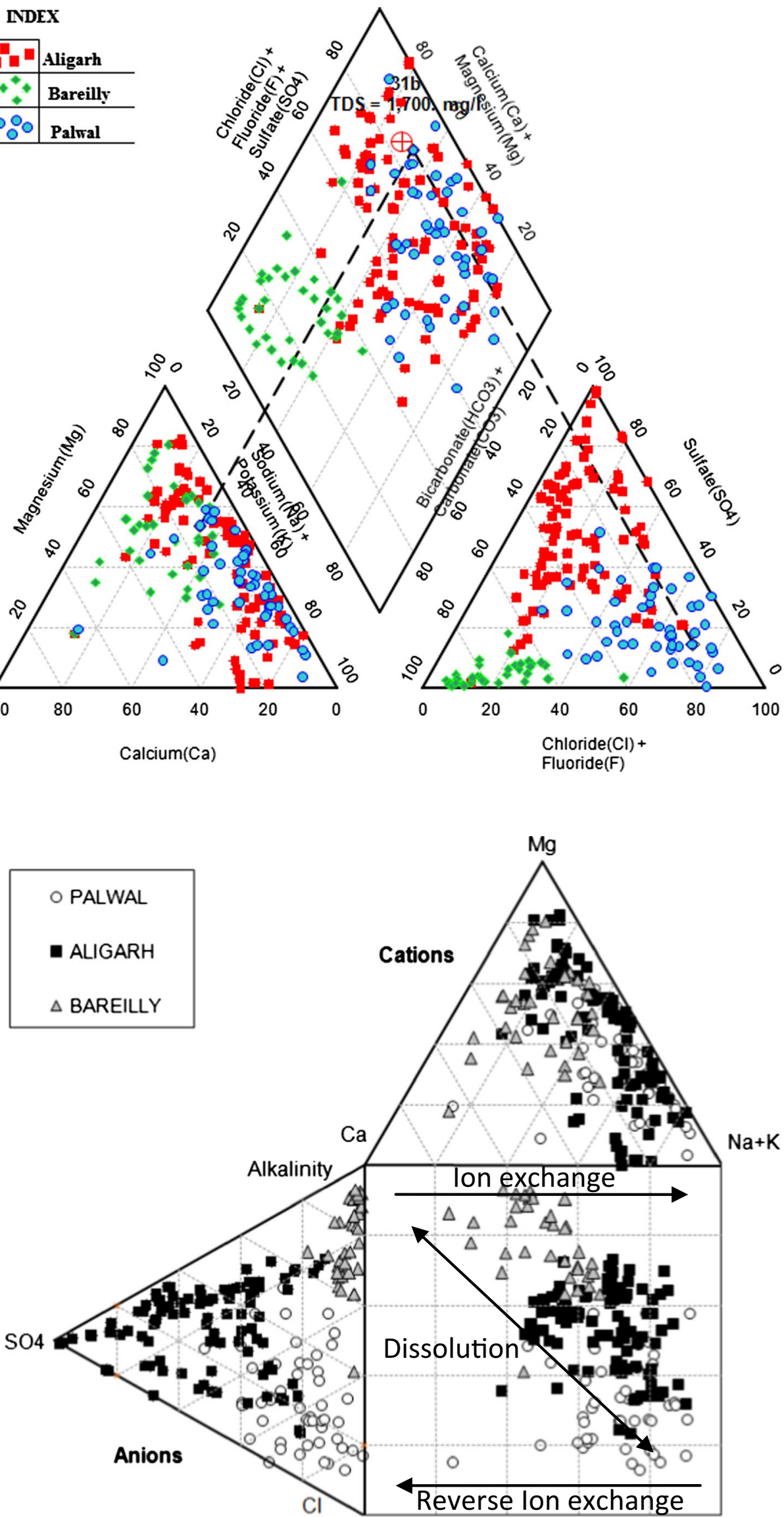


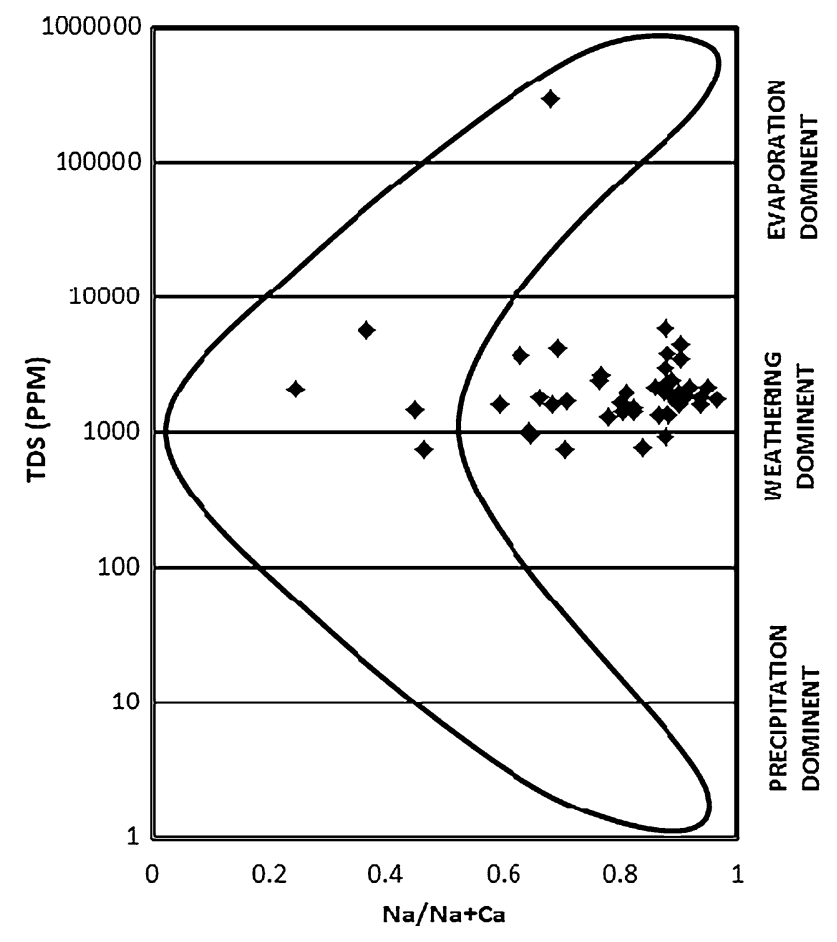

Fig. 7 Fig Variation of weight ratio of $\mathrm{Na}^{+} /\left(\mathrm{Na}^{+}+\mathrm{Ca}^{+2}\right)$ as a function of TDS(after Gibbs 1970) is associated with $\mathrm{pH}, \mathrm{SO}_{4}^{-2}$ and $\mathrm{Mg}^{+2}$ probably contributed by the dissolution of $\mathrm{MgSO}_{4}$ and subsequent change in $\mathrm{pH}$. The third component is associated with $\mathrm{Na}^{+}$and TDS as a proxy to strong evapotranspiration. The fourth component explains about $8 \%$ of the total variance that is associated with only $\mathrm{HCO}_{3}{ }^{-}$.

The first four PCs during post-monsoon explain the less total variance than pre-monsoon. The first component explains $29 \%$ of the total variance and is associated with hardness, $\mathrm{HCO}_{3}{ }^{-}, \mathrm{F}^{-}$and TDS. The second component is associated with $\mathrm{pH}, \mathrm{Na}^{+}$and $\mathrm{K}^{+}$. The third component includes $\mathrm{Na}^{+}$and $\mathrm{NO}_{3}{ }^{-}$in association with inverse relationships to the second component. It indicates that leaching results in an increase in $\mathrm{NO}_{3}{ }^{-}$and decrease in $\mathrm{Na}^{+}$concentration in groundwater during monsoonal recharge. The fourth component is related to $\mathrm{SO}_{4}^{-2}, \mathrm{Cl}^{-}$and $\mathrm{Mg}^{+2}$ as dissolution of $\mathrm{Mg}$ salts during monsoonal recharge.

\section{Drinking water quality analysis}

The suitability of the groundwater has been assessed for drinking purposes using BIS (2012) standards. It is found that most of the samples have a high degree of magnesium content. Undesirably high $\mathrm{SO}_{4}^{-2}, \mathrm{Cl}^{-}, \mathrm{EC}, \mathrm{F}^{-}$and $\mathrm{Ca}^{+2}$ values were found in $22 \%, 18 \%, 10 \%, 8 \%$ and $3 \%$ of the
Fig. 8 Scatter diagram showing (I) $\left(\mathrm{Na}^{+}+\mathrm{K}^{+}\right)$versus total cation (II) $\left(\mathrm{Ca}^{+2}+\mathrm{Mg}^{+2}\right)$ versus $\mathrm{HCO}_{3}^{-}$(III) $\left(\mathrm{Ca}^{+2}+\mathrm{Mg}^{+2}\right)$ versus $\left(\mathrm{Na}^{+}+\mathrm{K}^{+}\right)(\mathrm{IV})$ $\left(\mathrm{Ca}^{+2}+\mathrm{Mg}^{+2}\right)$ versus total cations
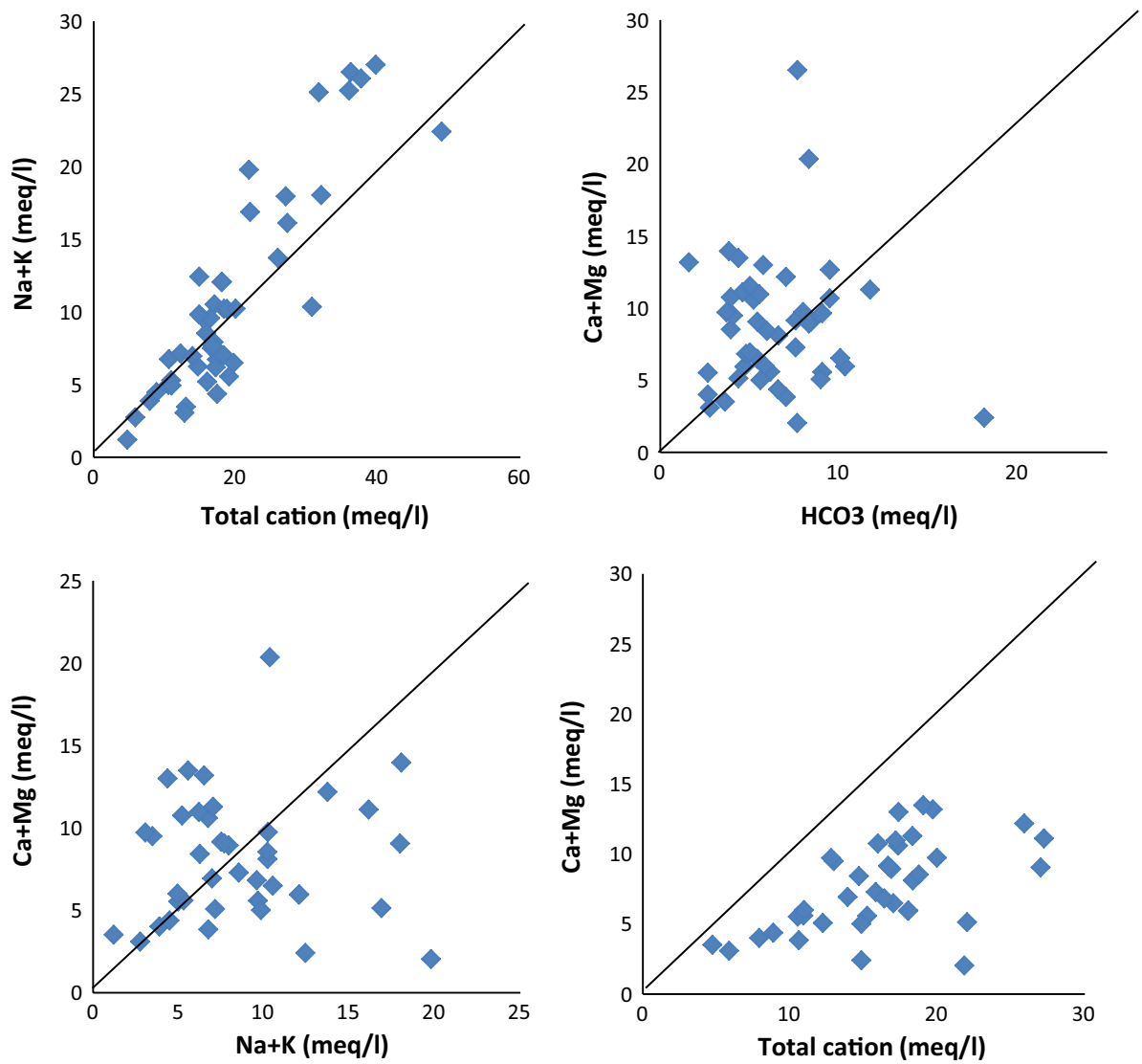
samples, respectively. It is observed that region of bad water quality lies in highly urbanized areas and localities along the Yamuna River. That is heavily affected by agriculture activities assisted by dense canal network system along with the industrial activity. High fluoride content was observed in the northwest and southeast side of the district dominated by the metamorphic rock of Aravalli Mountains and low lying region of Yamuna River. The difference in nitrate concentration in the post- and premonsoon indicates higher values near city due to leaching from sewage and intense fertilizers-supported vegetable crops near city areas. But in the south of the district this fluctuation is negative that indicates the utilization of enhanced nitrate in the pre-monsoon by the pre-monsoon crop.

The water quality index for the groundwater samples was determined to rank these samples from the drinking purpose. The water quality index provides an inclusive interpretation of the quality of surface and groundwater and its suitability for drinking purposes. The main purpose of WQI is to change the complex water quality data into understandable and usable information by which common people can know the status of water sources in a particular region (Akoteyon et al. 2011; Vasanthavigar et al. 2010). The weighted arithmetic index method is used in the present case for the calculation of WQI using 11 water quality characteristics, namely turbidity, $\mathrm{pH}$, total hardness, bicarbonate, chloride, total dissolved solids, calcium, magnesium, sulfate, nitrate and fluoride. A detailed distribution of WQI suggests that $13 \%$ fall in good category, $31 \%$ and $34 \%$ of the total water samples were found with poor and very poor water quality standard, $21 \%$ fall under the very poor category and $3 \%$ samples are unsuitable water quality standard (Table 7).

\section{Irrigation water quality analysis}

The groundwater is also being heavily used for irrigation purposes in the area due to the absence of large surface bodies. In view of irrigation utility, the groundwater samples were also analyzed for their suitability and usefulness to meet the irrigational needs of farmers. The suitability of water for irrigation purposes has been evaluated through three parameters, namely SAR (sodium adsorption ratio), sodium percent ( $\mathrm{Na} \%$ ) and $\mathrm{RSC}$ (residual sodium carbonate).
Table 5 Matrix of correlation for the hydrochemical parameters in groundwater

\begin{tabular}{|c|c|c|c|c|c|c|c|c|c|c|c|c|}
\hline & $p H$ & $E C$ & $\mathrm{HCO}_{3}^{-}$ & $\mathrm{SO}_{4}^{2-}$ & $\mathrm{NO}_{3}^{-}$ & $\mathrm{Cl}^{-}$ & $F^{-}$ & $\mathrm{Na}^{+}$ & $K^{+}$ & $\mathrm{Ca}^{+2}$ & $\mathrm{Mg}^{+2}$ & $T D S$ \\
\hline \multicolumn{13}{|c|}{ Post-monsoon 2016} \\
\hline $\mathrm{pH}$ & 1.00 & & & & & & & & & & & \\
\hline $\mathrm{EC}$ & 0.25 & 1.00 & & & & & & & & & & \\
\hline $\mathrm{HCO}_{3}^{-}$ & 0.27 & 0.92 & 1.00 & & & & & & & & & \\
\hline $\mathrm{SO}_{4}^{2-}$ & 0.01 & 0.70 & 0.47 & 1.00 & & & & & & & & \\
\hline $\mathrm{NO}_{3}^{-}$ & 0.14 & 0.05 & -0.08 & 0.10 & 1.00 & & & & & & & \\
\hline $\mathrm{Cl}^{-}$ & 0.02 & 0.78 & 0.58 & 0.62 & 0.04 & 1.00 & & & & & & \\
\hline $\mathrm{F}^{-}$ & 0.29 & 0.08 & 0.09 & 0.02 & 0.23 & -0.11 & 1.00 & & & & & \\
\hline $\mathrm{Na}^{+}$ & 0.01 & 0.85 & 0.77 & 0.65 & 0.00 & 0.87 & 0.00 & 1.00 & & & & \\
\hline $\mathrm{K}^{+}$ & 0.37 & 0.59 & 0.39 & 0.52 & 0.61 & 0.48 & 0.27 & 0.43 & 1.00 & & & \\
\hline $\mathrm{Ca}^{+2}$ & 0.17 & 0.59 & 0.50 & 0.17 & 0.06 & 0.44 & 0.07 & 0.41 & 0.34 & 1.00 & & \\
\hline $\mathrm{Mg}^{+2}$ & 0.19 & 0.65 & 0.42 & 0.81 & -0.01 & 0.57 & 0.02 & 0.49 & 0.46 & 0.06 & 1.00 & \\
\hline TDS & 0.25 & 1.00 & 0.92 & 0.70 & 0.05 & 0.78 & 0.08 & 0.85 & 0.59 & 0.59 & 0.65 & 1.00 \\
\hline \multicolumn{13}{|c|}{ Pre-monsoon 2017} \\
\hline $\mathrm{pH}$ & 1.00 & & & & & & & & & & & \\
\hline $\mathrm{EC}$ & -0.50 & 1.00 & & & & & & & & & & \\
\hline $\mathrm{HCO}_{3}^{-}$ & -0.40 & 0.87 & 1.00 & & & & & & & & & \\
\hline $\mathrm{SO}_{4}^{2-}$ & 0.18 & 0.03 & 0.00 & 1.00 & & & & & & & & \\
\hline $\mathrm{NO}_{3}^{-}$ & -0.24 & 0.30 & 0.09 & -0.09 & 1.00 & & & & & & & \\
\hline $\mathrm{Cl}^{-}$ & -0.38 & 0.83 & 0.53 & -0.02 & 0.17 & 1.00 & & & & & & \\
\hline $\mathrm{F}^{-}$ & -0.14 & 0.21 & 0.31 & -0.04 & 0.03 & 0.09 & 1.00 & & & & & \\
\hline $\mathrm{Na}^{+}$ & -0.51 & 0.93 & 0.71 & 0.07 & 0.27 & 0.84 & 0.16 & 1.00 & & & & \\
\hline $\mathrm{K}^{+}$ & -0.48 & 0.71 & 0.55 & -0.05 & 0.37 & 0.56 & 0.02 & 0.57 & 1.00 & & & \\
\hline $\mathrm{Ca}^{+2}$ & -0.19 & 0.31 & 0.28 & -0.27 & 0.11 & 0.40 & 0.10 & 0.16 & 0.15 & 1.00 & & \\
\hline $\mathrm{Mg}^{+2}$ & -0.21 & 0.33 & 0.13 & -0.03 & 0.34 & 0.25 & -0.13 & 0.37 & 0.44 & -0.50 & 1.00 & \\
\hline TDS & -0.50 & 1.00 & 0.87 & 0.03 & 0.30 & 0.83 & 0.21 & 0.93 & 0.71 & 0.31 & 0.33 & 1.00 \\
\hline
\end{tabular}


Table 6 Principal component analysis (PCA) of groundwater quality parameters

\begin{tabular}{|c|c|c|c|c|c|c|c|c|}
\hline & \multicolumn{4}{|c|}{ Pre-monsoon 2017} & \multicolumn{4}{|c|}{ Post-monsoon 2016} \\
\hline & Factor 1 & Factor 2 & Factor 3 & Factor 4 & Factor 1 & Factor 2 & Factor 3 & Factor 4 \\
\hline Hardness & 0.86 & -0.20 & 0.02 & 0.01 & 0.56 & -0.33 & 0.04 & 0.16 \\
\hline $\mathrm{pH}$ & -0.16 & 0.72 & -0.46 & -0.07 & -0.12 & 0.87 & -0.11 & 0.00 \\
\hline $\mathrm{HCO}_{3}{ }^{-}$ & -0.03 & -0.04 & -0.17 & 0.85 & 0.91 & 0.02 & 0.28 & 0.26 \\
\hline $\mathrm{SO}_{4}^{-2}$ & 0.05 & 0.88 & 0.20 & 0.01 & -0.01 & 0.45 & 0.12 & 0.69 \\
\hline $\mathrm{NO}_{3}^{-}$ & 0.87 & 0.19 & -0.02 & -0.15 & 0.44 & 0.43 & 0.51 & -0.25 \\
\hline $\mathrm{Cl}^{-}$ & 0.50 & 0.53 & -0.19 & 0.05 & 0.28 & -0.09 & 0.34 & 0.66 \\
\hline $\mathrm{F}^{-}$ & 0.90 & -0.05 & 0.12 & -0.04 & 0.82 & -0.29 & 0.01 & 0.07 \\
\hline $\mathrm{Na}^{+}$ & -0.04 & -0.02 & 0.85 & -0.18 & 0.14 & 0.55 & -0.58 & 0.12 \\
\hline $\mathrm{K}^{+}$ & 0.61 & -0.37 & 0.21 & 0.28 & 0.19 & -0.67 & -0.08 & -0.03 \\
\hline $\mathrm{Ca}^{+2}$ & 0.48 & -0.10 & -0.44 & -0.44 & 0.09 & 0.00 & 0.78 & 0.29 \\
\hline $\mathrm{Mg}^{+2}$ & 0.74 & 0.56 & -0.14 & -0.06 & 0.43 & -0.19 & -0.23 & 0.63 \\
\hline TDS & 0.16 & -0.09 & 0.87 & 0.00 & 0.79 & 0.01 & -0.30 & 0.00 \\
\hline Eigenvalues & 4.65 & 2.20 & 2.08 & 1.07 & 3.77 & 2.13 & 1.65 & 1.62 \\
\hline$\%$ of Variance & 35.80 & 16.94 & 16.01 & 8.20 & 28.99 & 16.41 & 12.71 & 12.47 \\
\hline$\%$ Cumulative variance & 35.80 & 52.74 & 68.74 & 76.95 & 28.99 & 45.40 & 58.11 & 70.58 \\
\hline
\end{tabular}

\begin{tabular}{|c|c|c|c|c|}
\hline Parameter & References & Range & Class & No. of samples \\
\hline \multirow[t]{5}{*}{$\% \mathrm{Na}$} & \multirow[t]{5}{*}{ Wilcox (1955) } & $<20$ & Excellent & 18 \\
\hline & & $20-40$ & Good & 21 \\
\hline & & $40-60$ & Permissible & 6 \\
\hline & & $60-80$ & Doubtful & None \\
\hline & & $>80$ & Unsuitable & None \\
\hline \multirow[t]{4}{*}{ SAR } & \multirow[t]{4}{*}{ Richard (1954) } & $<10$ & Excellent (S1) & 58 \\
\hline & & $10-18$ & Good (S2) & 1 \\
\hline & & $18-26$ & Doubtful (S3) & None \\
\hline & & $>26$ & Unsuitable (S4) & None \\
\hline \multirow[t]{3}{*}{ RSC } & \multirow[t]{3}{*}{ Richard (1954) } & $<1.25$ & Good & \\
\hline & & $1.25-2.5$ & Doubtful & 18 \\
\hline & & $>2.5$ & Unsuitable & 15 \\
\hline \multirow[t]{2}{*}{ TDS } & \multirow[t]{2}{*}{ Salton (1999) } & $<1000 \mathrm{mg} / \mathrm{l}$ & Fresh & 6 \\
\hline & & $>1000 \mathrm{mg} / \mathrm{l}$ & Brackish & 41 \\
\hline \multirow[t]{2}{*}{$\mathrm{Cl}$} & \multirow[t]{2}{*}{ Salton (1999) } & $<15 \mathrm{meq} / \mathrm{l}$ & Normal & 17 \\
\hline & & $>15$ meq/l & Contaminated & 30 \\
\hline \multirow[t]{2}{*}{$\mathrm{SO}_{4}^{-2}$} & \multirow[t]{2}{*}{ Salton (1999) } & $<6$ meq/l & Normal & 30 \\
\hline & & $>6$ meq/l & Contaminated & 17 \\
\hline \multirow[t]{2}{*}{$\mathrm{HCO}_{3}^{-1}$} & \multirow[t]{2}{*}{ Salton (1999) } & $2-7 \mathrm{meq} / \mathrm{l}$ & Normal & 16 \\
\hline & & $>7 \mathrm{meq} / \mathrm{l}$ & Contaminated & 31 \\
\hline \multirow[t]{5}{*}{ WQI value } & & $0-25$ & Excellent & None \\
\hline & & $26-50$ & Good & 6 \\
\hline & & $51-75$ & Poor & 15 \\
\hline & & $75-100$ & Very poor & 16 \\
\hline & & $>100$ & Unsuitable & 10 \\
\hline
\end{tabular}

Table 7 Classification of groundwater samples in Palwal District

\section{Sodium Adsorption Ratio (SAR)}

Sodium adsorption ratio is used to evaluate the excess of sodium with calcium and magnesium (Richards 1954). In general, the permeability of soil reduces due to excessive sodium content in irrigation water. The use of water having a high SAR level continuously can lead to the increase 
in $\mathrm{Na} \%$ level over the time, which in turn can adversely affect soil infiltration and percolation rates. Fine-textured soils, especially those high in clay, are most subject to this action. Certain amendments may be required to maintain soils under high SARs. Calcium and magnesium, if present in the soil in large enough quantities, will counter the effects of sodium and help to maintain good soil properties (Fipps 2003). In addition to this, the excessive SAR levels of irrigation water can also cause soil crusting, poor seedling and poor aeration (Lesch and Suarez 2009). The SAR of groundwater samples is calculated, and the results of SAR were found within the range $0.8-17.85$ with an average value of 3.6. Based on classification (Richards 1954), it is observed that the groundwater of the study area was excellent and good for irrigation purposes. The Wilcox diagram has been prepared to determine the suitability of the groundwater for irrigation purposes (Fig. 9). It is showed that $62 \%$ of the samples belong to good water category (C3S1, C2S1), 14\% samples belong to moderate water category (C3S2, C3S3), 7\% samples fall under bad category (C4S3, C4S2) and 5\% samples fall under very bad quality category (C4S4).

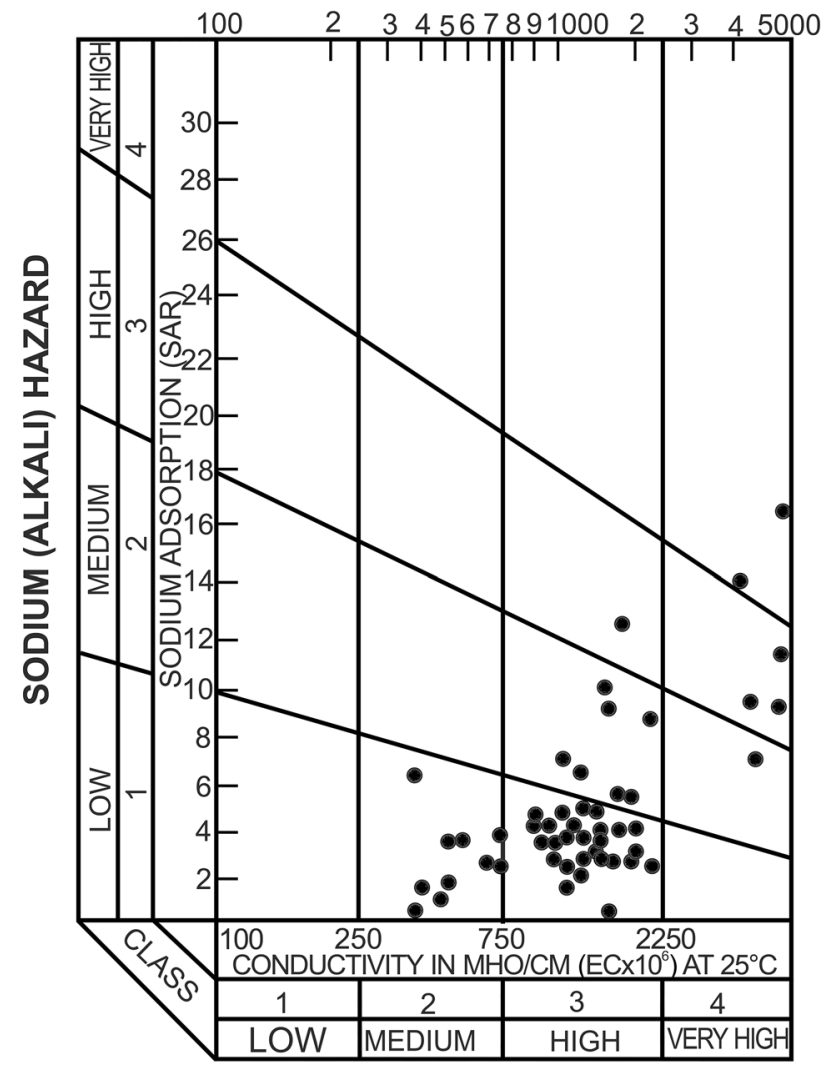

SALINITY HAZARD

Fig. 9 Wilcox diagram representing the groundwater quality for irrigation purposes

\section{Sodium Percentage ( $\mathrm{Na} \%)$}

Sodium percentage is a parameter to evaluate the water quality for irrigation purposes. Excess of sodium in water reacts with soil, reduces soil permeability and supports little or no plant growth (Wilcox 1955). $\mathrm{Na}^{+} \%$ in groundwater ranged from 9 to $60 \%$ with an average of $25 \%$. The calculated $\mathrm{Na} \%$ shows that $87 \%$ of all samples fall within the excellent/good for irrigation category and $12.5 \%$ fall under permissible category (Table 7).

\section{Residual sodium carbonate (RSC)}

The sodium hazard also increases, if the water contains a higher concentration of bicarbonate ions. As the soil solution becomes more concentrated, there is a tendency for calcium and magnesium to precipitate as carbonates, thereby increasing the relative proportion of sodium as a consequence. In the present case, RSC was used to quantify the effect of the carbonate and bicarbonate (Eaton 1950) as RSC. Distribution of samples based on RSC value indicated that the majority of the samples fall under doubtful and unsuitable categories for irrigation purposes.

\section{Impact of land use/land cover change on groundwater depth}

A rapid increase in population has been observed from 59,168 in 1991 and 131, 926 in 2011 with the $122 \%$ growth (Census of India 1991, 2011). During this period, the settlement area of the main Palwal City has been increased by 177\% (Fig. 10). The increased population and associated services withdraw water from local aquifer, resulting in a decline of groundwater level. Time series of the groundwater depths were assessed using the linear regression line method at few locations. The average declining trends of the groundwater level vary from 0.10 to $0.40 \mathrm{~m} / \mathrm{year}$, and the spatial trends show minimum decline near the Yamuna flood plain and maximum decline near the Palwal City (Fig. 11). However, the areas near Bhagola village along the canal show a decrease in groundwater depth with a rate of $0.05 \mathrm{~m} / \mathrm{year}$. The declining groundwater depth will result in decreasing well productivity in the near future. Deep groundwater is normally associated with solute-enriched groundwater, and this will be further detrimental to the suitability of groundwater for irrigation, drinking and other purposes.

\section{Conclusions}

Groundwater in the study area is characterized by deep origin as revealed by meteoric genesis index $R_{2}$. The source of chemical in groundwater is mainly controlled by rock 
Fig. 10 Urbanization/urban sprawling and growth of Palwal City

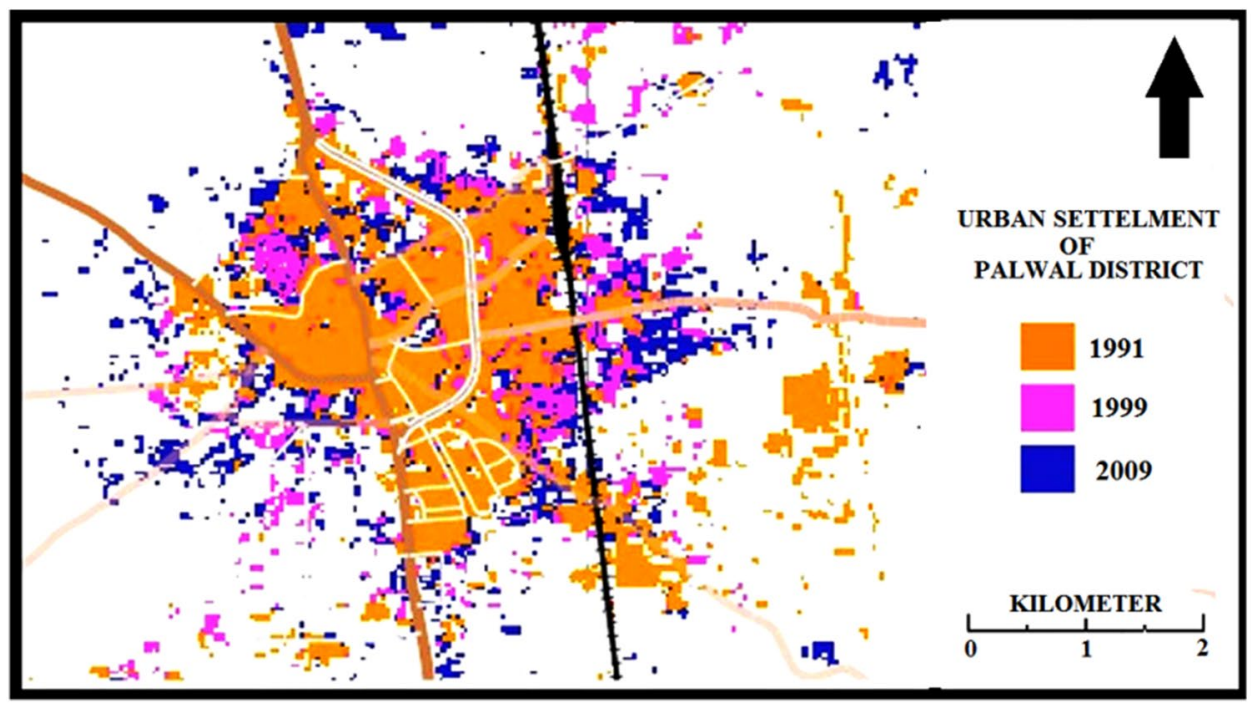

Fig. 11 Groundwater fluctuation pattern in Palwal District

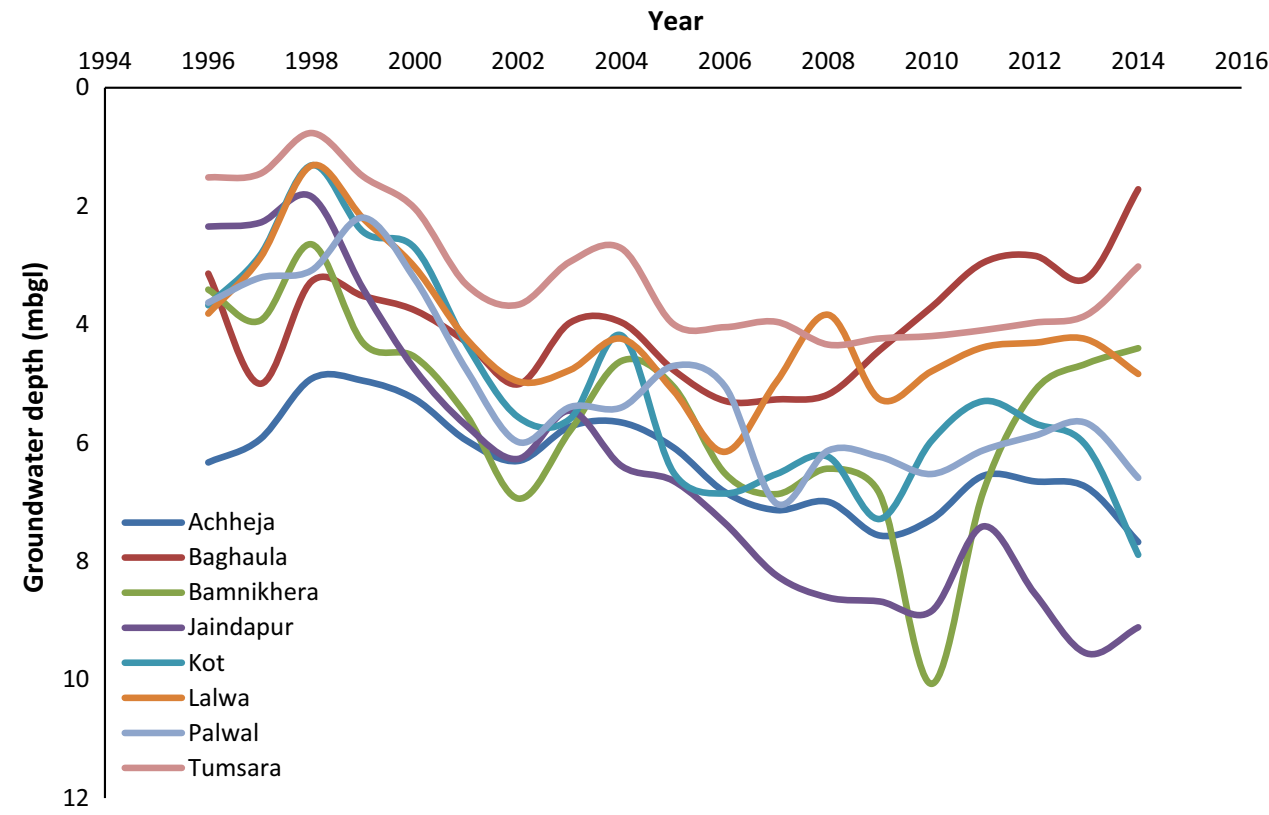

weathering and evaporation processes. The major portion of ions in groundwater is derived by combined weathering of carbonate and silicate rocks. Durov diagram indicated that dissolution and reverse ion exchange are the major processes that control the hydrochemistry. Spatial variation in the contribution of ion exchange, dissolution and reverse exchange processes is mainly controlled by climatic conditions in the Ganga basin.

Spatial distribution of the fluoride is controlled by rock type and low lying areas, while high $\mathrm{Mg}^{+2}$ and $\mathrm{SO}_{4}^{-2}$ distribution is associated with urban and peri-urban areas. Groundwater quality index suggested that a fair percentage of water samples fall under the category of very poor (34\%) and unsuitable (21\%) for drinking purposes. Most of the samples are suitable for irrigation purposes on the basis of SAR. However, continuous usage of groundwater with high TDS with moderate Na\% may result in the development of salinity in soil.

Acknowledgments The authors would like to express their thanks to Chairman, Department of Geology, Aligarh Muslim University, for providing the laboratory and library facilities. The authors would like to appreciate the financial assistance in the form of Rajiv Gandhi fellowship for $\mathrm{PhD}$ research student provided by UGC, Ministry of HRD, Govt of India, to conduct the present research work.

Open Access This article is licensed under a Creative Commons Attribution 4.0 International License, which permits use, sharing, 
adaptation, distribution and reproduction in any medium or format, as long as you give appropriate credit to the original author(s) and the source, provide a link to the Creative Commons licence, and indicate if changes were made. The images or other third party material in this article are included in the article's Creative Commons licence, unless indicated otherwise in a credit line to the material. If material is not included in the article's Creative Commons licence and your intended use is not permitted by statutory regulation or exceeds the permitted use, you will need to obtain permission directly from the copyright holder. To view a copy of this licence, visit http://creativecommons .org/licenses/by/4.0/.

\section{References}

Ahmad S, Mazhar SN (2020) Hydrochemical characteristics, groundwater quality and sources of solute in the Ramganga Aquifer, Central Ganga Plain, Bareilly District, Uttar Pradesh. J Geol Soc India (In Progress: Accepted)

Akbal F, Gürel L, Bahadır T, Güler İ, Bakan G, Büyükgüngör H (2011) Water and sediment quality assessment in the mid-Black Sea coast of Turkey using multivariate statistical techniques. Environ Earth Sci 64:1387-1395

Akoteyon IS, Mbata UA, Olalude GA (2011) Investigation of heavy metal contamination in groundwater around landfill site in a typical sub-urban settlement in Alimosho Lagos-Nigeria. J Appl Sci Environ. 6(2):155-163

APHA (1998) Standard Methods for the Examination of Water and Wastewater (1998), 20th edn. American Public Health Association, Washington, DC, p 2200

BIS (2012) Bureau of Indian Standards)-Drinking water quality 10500 , Indian Standard Drinking Water Specification. BIS, New Delhi, pp 1-8

Brindha K, Elango L (2010) Study on bromide in groundwater parts of Nalgonda district, Andhra Pradesh. Earth Sci India 3:73-80

Census of India (1991) Registrar General and Census Commissioner of India under the Ministry of Home Affairs, Government of India

Census of India (2011), Registrar General and Census Commissioner of India under the Ministry of Home Affairs, Government of India

CGWB (2006) Hydrogeology of Faridabad district with special reference to municpla corporation area Faridabad Haryana state, CGWB report, Chandigarh

CGWB (2013) Aquifer mapping and management plan, NCR (Natianal Captital Region), Haryana, volume-1, pp 231

Department of Town and country Planning (2013) District Disaster Management Plan Palwal. Govermnent of Haryana, Department of Revenue and Disater Management

Eaton FM (1950) Significant of carbonates in irrigation waters. Soil Sci 69:123-133

Fipps G (2003) Irrigation water quality standards and salinity management strategies. Texas Agricultural Extension Service, Texas A\&M University System, College Station, TX (USA). B-1667, 4-03, pp 1-19

Gibbs RJ (1970) Mechanisms controlling world water chemistry. Science 17:1088-1090

Gupta S, Mahato A, Roy P, Datta JK, Saha RN (2008) Geochemistry of groundwater, Burdwan district, West Bengal, India. Environ Geol 53:1271-1282

Irfan M, Said M (2008) Hydrochemical characteristics and the effects of irrigation on groundwater quality in Harran Plain, GAP Project, Turkey. Environ Geol 54:183-196
Jalali M (2006) Chemical characteristics of groundwater in parts of mountainous region, Alvand, Hamadan, Iran. Environ Geol $51: 433-446$

Jalali M (2007) Assessment of the chemical components of Famenin groundwater, western Iran. Environ Geochem Health 29:357-374

Kumar M, Kumari K, Singh UK, Ramananthan AL (2009) Hydrogeochemical processes in the groundwater environment of Muktsar, Punjab: conventional graphical and multivariate statistical approach. Environ Geol 57:873-884

Kumar M, Rao MS, Kumar B, Ramanathan AL (2011) Identification of aquifer-recharge zones and sources in an urban development area (Delhi, India), by correlating isotopic tracers with hydrological features. Hydrogeol J 19(2):463-474

Lesch SM, Suarez DL (2009) A short note on calculating the adjusted SAR Index. Am Soc Agric Biol Eng 52(2):493-496

NBSS and LUP (1993) Soil Map of Haryana, -National Bureau of Soil Survey and Land Use Planning, Amravati Road, Nagpur 440 033, India

Piper, AM (1953) A graphic procedure in the geochemical interpretation of water analysis. Washington D.C: United States Geological Survey. OCLC 37707555 . ASIN B0007HRZ36

Ramesh K, Elango L (2005) Groundwater quality assessment in Tondiar basin. Ind J Environ Prot 26(6):497-504

Ramesh K, Vennila S (2012) Hydrochemical analysis and evaluation of groundwater quality in and around Hosur, Krishnagiri District, Tamil Nadu, India. Int J Res Chem Environ 2(3):113-122

Richards LA (1954) Diagnosis and improvement of saline and alkali soils. Agriculture Handbook 60, US DA, Washington, DC

Soltan ME (1999) Evaluation of groundwater quality in Dakhla Oasis (Egyptian Western desert). Environ Monit Assess 57:157-168

Srinivasamoorthy K, Vijayaraghavan K, Vasanthavigar M, Sarma VS, Chidambaram S, Anandhan P (2010) Assessment of groundwater quality with special emphasis on fluoride contamination in crystalline bed rock aquifers of Mettur region, Tamilnadu, India. Arab J Geosci 5:83-94

Stallard RF, Edmond JM (1983) Geochemistry of the Amazon: 2. The Influence of Geology and Weathering Environment on the Dissolved Load. J Geophys Res Oceans 88:9671-9688

Varol S, Davraz A (2014) Assessment of geochemistry and hydrogeochemical processes in groundwater of the Tefenni plain (Burdur/ Turkey). Environ Earth Sci 71(11):4657-4673

Vasanthavigar M, Srinivasamoorthy K, Vijayaragavan K, Rajiv Ganthi R, Chidambaram S, Anandhan P, Manivannan R, Vasudevan S (2010) Application of water quality index for groundwater quality assessment: Thirumanimuttar sub-basin, Tamilnadu, India. Environ Monit Assess 171(1-4):595-609

Vasanthavigar M, Srinivasamoorthy K, Ganthi R, Vijayaraghavan K, Sarma VS (2012) Characterisation and quality assessment of groundwater with a special emphasis on irrigation utility: Thirumanimuttar sub-basin, Tamil Nadu, India. Arab J Geosci 5:245-258

Wasim SM, Kursheed S, Shah A, Raghuvanshi D (2014) Groundwater Quality in Parts of Central Ganga Basin. Aligarh City, Proc Indian Natn Sci Acad 80:123-142

Wilcox LV (1955) Classification and use of irrigation waters. USDA Circular No. 969, p 19

Zhang L, Song X, Xia J, Yuan R, Zhang Y, Liu X, Han D (2011) Major element chemistry of the Huai River basin, China. Appl Geochem 26:293-300

Publisher's Note Springer Nature remains neutral with regard to jurisdictional claims in published maps and institutional affiliations. 